Linköping studies in science and technology. Thesis.

No. 1744

Licentiate's Thesis

\title{
Detection and Tracking in Thermal Infrared Imagery
}

\section{Amanda Berg}

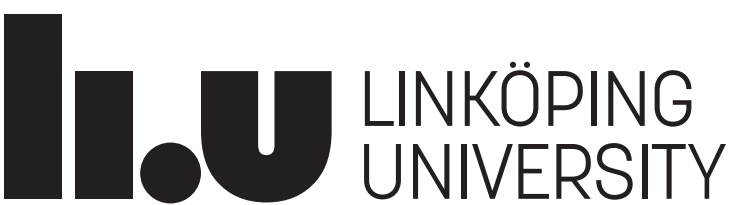


This is a Swedish Licentiate's Thesis.

Swedish postgraduate education leads to a Doctor's degree and/or a Licentiate's degree.

A Doctor's Degree comprises 240 ECTS credits (4 years of full-time studies).

A Licentiate's degree comprises 120 ECTS credits,

of which at least 60 ECTS credits constitute a Licentiate's thesis.

Linköping studies in science and technology. Thesis.

No. 1744

\title{
Detection and Tracking in Thermal Infrared Imagery
}

Amanda Berg

\author{
amanda.bergeliu.se \\ www.cvl.isy.liu.se \\ Computer Vision Laboratory \\ Department of Electrical Engineering \\ Linköping University \\ SE-581 83 Linköping \\ Sweden
}

ISBN 978-91-7685-789-2

ISSN 0280-7971

Copyright (C) 2016 Amanda Berg

Printed by LiU-Tryck, Linköping, Sweden 2016 


\section{Abstract}

Thermal cameras have historically been of interest mainly for military applications. Increasing image quality and resolution combined with decreasing price and size during recent years have, however, opened up new application areas. They are now widely used for civilian applications, e.g., within industry, to search for missing persons, in automotive safety, as well as for medical applications. Thermal cameras are useful as soon as it is possible to measure a temperature difference. Compared to cameras operating in the visual spectrum, they are advantageous due to their ability to see in total darkness, robustness to illumination variations, and less intrusion on privacy.

This thesis addresses the problem of detection and tracking in thermal infrared imagery. Visual detection and tracking of objects in video are research areas that have been and currently are subject to extensive research. Indications of their popularity are recent benchmarks such as the annual Visual Object Tracking (VOT) challenges, the Object Tracking Benchmarks, the series of workshops on Performance Evaluation of Tracking and Surveillance (PETS), and the workshops on Change Detection. Benchmark results indicate that detection and tracking are still challenging problems.

A common belief is that detection and tracking in thermal infrared imagery is identical to detection and tracking in grayscale visual imagery. This thesis argues that the preceding allegation is not true. The characteristics of thermal infrared radiation and imagery pose certain challenges to image analysis algorithms. The thesis describes these characteristics and challenges as well as presents evaluation results confirming the hypothesis.

Detection and tracking are often treated as two separate problems. However, some tracking methods, e.g. template-based tracking methods, base their tracking on repeated specific detections. They learn a model of the object that is adaptively updated. That is, detection and tracking are performed jointly. The thesis includes a template-based tracking method designed specifically for thermal infrared imagery, describes a thermal infrared dataset for evaluation of templatebased tracking methods, and provides an overview of the first challenge on shortterm, single-object tracking in thermal infrared video. Finally, two applications employing detection and tracking methods are presented. 



\section{Populärvetenskaplig sammanfattning}

Sensorer som kan mäta termisk infraröd strålning över ett större område och producera en visuell bild, så kallade värmekameror, har länge använts inom militären. Däremot har värmekameror inte varit lika vanliga för civila tillämpningar, främst på grund av att de har varit dyra och utrymmeskrävande. På senare år har utvecklingen gått framåt och allteftersom kamerorna blivit mindre och billigare har även bildkvalitén förbättrats avsevärt. Nu finns det till och med en liten värmekamera som man kan fästa på sin mobiltelefon. I takt med att värmekamerorna har blivit mindre och billigare så har fler civila tillämpningar vuxit fram. Till exempel har det blivit vanligt att använda värmekameror i industrin, för att söka efter försvunna personer, i säkerhetssystem i bilar, för att upptäcka bränder och i medicinska sammanhang, för att nämna några. Värmekameror kommer till användning så snart det finns en mätbar temperaturskillnad. Jämfört med kameror känsliga för synligt ljus är de fördelaktiga i många situationer eftersom de kan producera en bild även i totalt mörker och de gör mindre intrång på personlig integritet.

Den här avhandlingen behandlar områdena detektion och följning av intressanta objekt i termiskt infraröda bilder och video. Två områden som det forskas mycket på för visuella bilder som också är relevanta för många civila tillämpningar. En vanlig uppfattning är att bildanalys i termiskt infraröda bilder är identiskt med bildanalys i visuella gråskalebilder. Avhandlingen visar att föregående påstående inte stämmer. Så länge en metod designad för visuella bilder inte är beroende av färgattribut kan den appliceras på termiskt infrarött, men resultaten för olika metoder har visat sig variera beroende på om de används på visuella eller termiska sekvenser. Det vill säga, olika angreppssätt fungerar olika bra i de olika modulariteterna.

Detektion och följning behandlas ofta som två separata problem. Vissa följningsmetoder, så kallade modellbaserade följningsmetoder, baserar emellertid följningen på upprepade, specifika, detektioner, där en modell av objektet som följs uppdateras kontinuerligt. Det vill säga, detektion och följning sker beroende av varandra. Avhandlingen inkluderar en modellbaserad följningsmetod för termisk infraröd video, beskriver ett termiskt infrarött dataset för utvärdering av den här typen av metoder, ger en överblick av resultaten för den första tävlingen i modellbaserad följning i infraröda sekvenser samt presenterar två tillämpningar där detektion och följning används. 



\section{Acknowledgements}

First, and most important of all, this thesis is not a product of the work of a single person, but by many who have taken part in the discussions as the work has progressed. There are, however, a number of persons to whom I would like to give extra credit:

- My supervisors Michael Felsberg and Jörgen Ahlberg for interesting discussions, great guidance, and patience. I would especially like to thank Jörgen who encouraged and convinced me to continue my studies postgraduate and Michael for believing in me and allowing me to join the research group.

- Termisk Systemteknik AB that has given me the opportunity to experience the best of two worlds, academia and industry.

- All fellow PhD students and colleagues at the Computer Vision Laboratory who have accepted me even though I am one of those industrial people.

- My colleagues at Termisk Systemteknik AB for contributing with expert knowledge on thermal infrared sensors and imagery.

- Fellow partners and colleagues in the P5 and IPATCH projects.

- Friends and family for always supporting me and providing much needed distractions whenever needed. Especially Olov for his unconditional love, support, and patience during approaching deadlines. As a token of appreciation, I am hereby dedicating page 35 to you.

- Finally, thanks to our, at the time of writing, unborn baby who has provided a much appreciated company while writing this thesis, constantly reminding me of your presence by kicking my internals. The company was appreciated even though the kicking sometimes made it difficult to concentrate.

The research leading to this thesis has been funded by the Swedish Research Council through the project "Learning Systems for Remote Thermography", grant no. D0570301, as well as by the European Community Framework Programme 7, Privacy Preserving Perimeter Protection Project (P5), grant agreement no. 312784, and Intelligent Piracy Avoidance using Threat detection and Countermeasure Heuristics (IPATCH), grant agreement no. 607567.

Linköping, May 2016 Amanda Berg 



\section{Contents}

\section{Background Theory}

1 Introduction 3

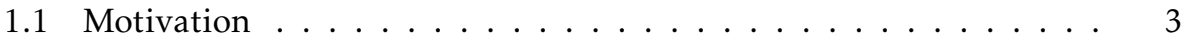

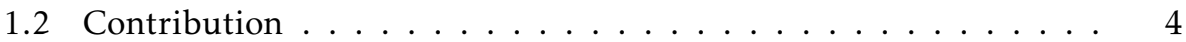

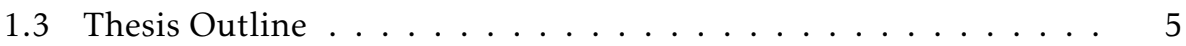

1.3.1 Outline Part I: Background Theory . . . . . . . . . . 5

1.3.2 Outline Part II: Included Publications . . . . . . . . . 5

1.4 Other Publications . . . . . . . . . . . . . . . . . . 9

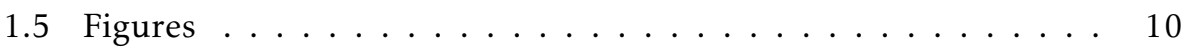

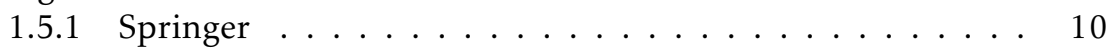

1.5 .2 IEEE ......................... 10

2 Thermal Infrared Imaging 11

2.1 Infrared and thermal radiation . . . . . . . . . . . . . 11

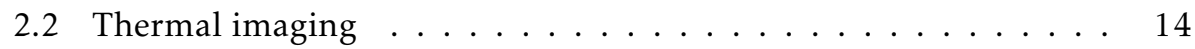

2.3 Advantages and limitations of thermal imaging . . . . . . . . 16

2.4 Image analysis in thermal infrared . . . . . . . . . . . 17

2.5 Applications ....................... 17

3 Detection and Tracking 21

3.1 Relation between detection, tracking, and classification . . . . . 21

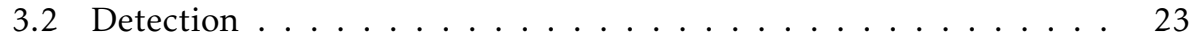

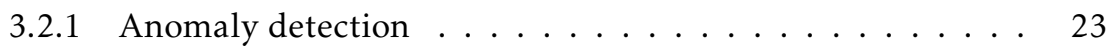

3.2.2 Detection of specific objects ................. 27

3.2.3 Detection in thermal infrared ............ 28

3.3 Tracking ........................... 30

3.3.1 Classes of tracking methods ............. 31

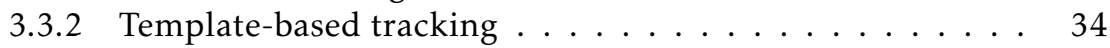

3.3 .3 Tracking metrics . . . . . . . . . . . . . 37

3.3.4 Tracking benchmarks and datasets .......... . . . 41

3.3.5 Tracking in thermal infrared . . . . . . . . . . . . 44 
3.4 Classification . . . . . . . . . . . . . . . . . . 47

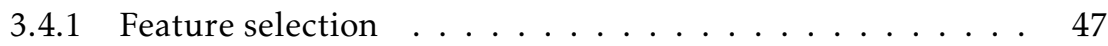

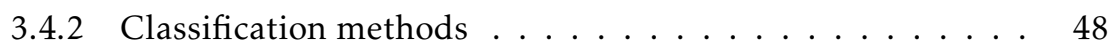

3.4.3 Classifier evaluation ............... 49

4 Concluding Remarks 53

4.1 Discussion . . . . . . . . . . . . . . . 53

4.2 Conclusions and future work . . . . . . . . . . . . . . 54

$\begin{array}{ll}\text { Bibliography } & 57\end{array}$

\section{Publications}

A A Thermal Object Tracking Benchmark $\quad 69$

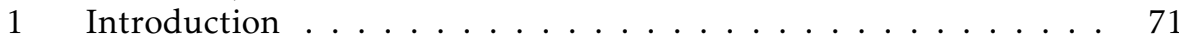

2 Background and motivation ................ 72

2.1 Why is TIR tracking different? . . . . . . . . 73

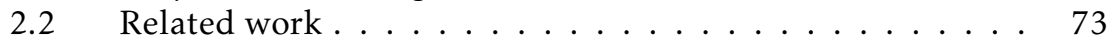

3 Description of the benchmark ................. 75

$3.1 \quad$ Dataset design criteria . . . . . . . . . . 75

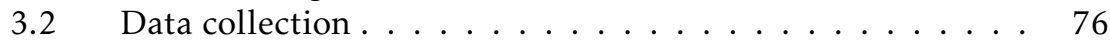

3.3 Included sequences . . . . . . . . . . . . . 76

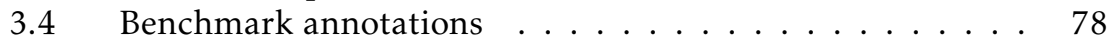

3.5 Evaluation methodology . . . . . . . . . . 78

4 Evaluation .................... 79

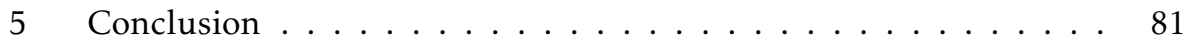

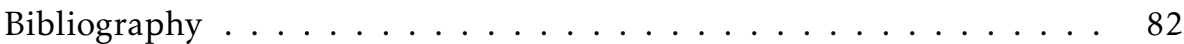

B The Thermal Infrared Visual Object Tracking VOT-TIR2015 Challenge Results $\quad 85$

1 Introduction . . . . . . . . . . . . . . 87

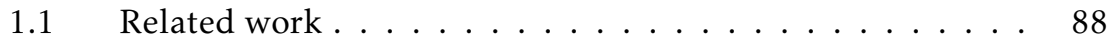

1.2 The VOT-TIR2015 challenge . . . . . . . . . . . 89

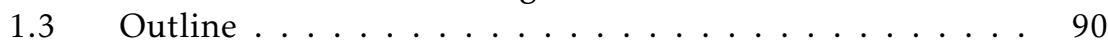

2 The VOT-TIR2015 dataset ................... 90

3 Performance measures and evaluation methodology . . . . . . . 91

4 Analysis and results . . . . . . . . . . . . . . . . . 91

4.1 The VOT2015 experiments . . . . . . . . . . . . . . . 91

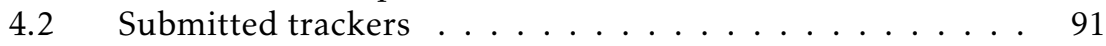

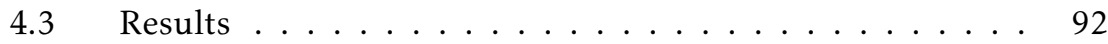

4.4 TIR-specific analysis and results . . . . . . . . . . . . 97

5 Conclusions ....................... 97

A Submitted trackers - VOT TIR . . . . . . . . . . . . . 98

A.1 Restore Point guided Kernelized Correlation Filters (KCFv2) 98

A.2 Scalable Kernel Correlation Filter with Sparse Feature Integration $(\mathrm{sKCF}) \ldots \ldots \ldots \ldots$ 
A.3 Motion-aware Complex Cell Tracker (MCCT) . . . . . . . 99

A.4 Point-based Kanade Lukas Tomasi color-Filter (PKLTF) . . 99

A.5 Struck .................... 100

A.6 Object-Aware Correlation Filter Tracker (OACF) . . . . . . 100

A.7 AOGTracker ................... 101

A.8 Geometric Structure Hyper-Graph based Tracker (G2T) . . 101

A.9 Scale-adaptive Multi-Expert Tracker (SME) . . . . . . . 102

A.10 NSAMF . . . . . . . . . . . . . . . 102

A.11 Edge Box Tracker (EBT) . . . . . . . . . . . 102

A.12 Multi-kernelized Correlation Filter plus (MKCF+) . . . . . 103

A.13 Clustering Correlation Tracking with Foreground Proposals (CCFP) . . . . . . . . . . . . . . . . . . . 103

A.14 SumShift . . . . . . . . . . . . . . 103

A.15 Spatially Regularized Discriminative Correlation Filter Tracker for IR (SRDCFir) . . . . . . . . . . . . . . . . . . . 104

A.16 Layered Deformable Parts tracker (LDP) . . . . . . . . . . . 104

A.17 Adaptive object region and Background weighted scaled Channel coded Distribution field tracker (ABCD) . . . . . . 105

A.18 Multi-Channel Multiple-Instance-Learning Tracker (CMIL) 105

A.19 DTracker .................... 105

A.20 simplified Proposal Selection Tracker (sPST) . . . . . . . 106

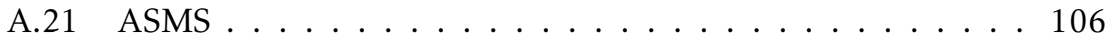

A.22 Flock of Trackers (FoT) . . . . . . . . . . . . 106

A.23 Spatio-temporal context tracker (STC) . . . . . . . . . 106 Bibliography ...................... 108

7 Channel Coded Distribution Field Tracking for Thermal Infrared Imagery

Introduction . . . . . . . . . . . . . 117

Thermal imaging and tracking . . . . . . . . . . . . . 117

Related Work . . . . . . . . . . . . . . . . . . 118

Proposed tracking method . . . . . . . . . . . . . 120

.1 Background contamination in tracking . . . . . . . . 120

.2 Background weighted model update (B-EDFT) . . . . . . . 121

.3 Adaptive object region (A-EDFT) . . . . . . . . . . . . . . . . . . . . . 122

.4 Scale change estimation . . . . . . . . . . . 122

.5 Combining the three methods (ABCD) . . . . . . . . . . . 124

.6 Combining with a detector for initialization . . . . . . . . 124

Evaluation and results . . . . . . . . . . . . . . 125

.1 Evaluation methodology . . . . . . . . . . . 125

.2 Experiments .................... 126

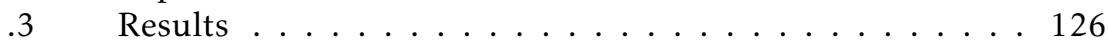

.4 Discussion ...................... 128

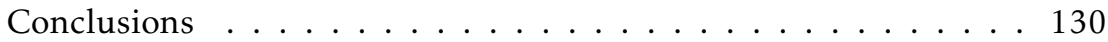


8 Detecting Rails and Obstacles using a Train-Mounted Thermal Camera

Introduction . . . . . . . . . . . . . . . . 141

.1 Railway vs. road detection . . . . . . . . . . . . . . 141

.2 Outline ....................... 142

Data collection ....................... 142

Proposed method . . . . . . . . . . . . . . . . 143

.1 System overview . . . . . . . . . . . . . 143

.2 Scene geometry and rail detection . . . . . . . . . . . 144

.3 Combined correction and anomaly detection . . . . . . 146

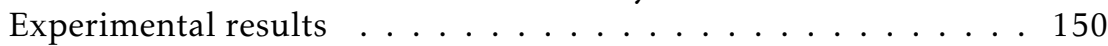

Conclusion . . . . . . . . . . . . . . . 152

$\begin{array}{ll}\text { Bibliography } & 153\end{array}$

9 Enhanced Analysis of Thermographic Images for Monitoring of District Heat Pipe Networks

Introduction . . . . . . . . . . . . . . . . 159

.1 Related work . . . . . . . . . . . . . 159

.2 Contribution . . . . . . . . . . . . . 160

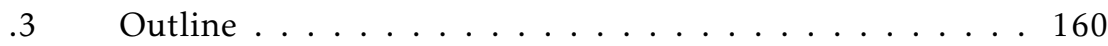

Data acquisition and leakage detection . . . . . . . . . 161

$.1 \quad$ Image data . . . . . . . . . . . . . . . 161

.2 GIS data . . . . . . . . . . . . . . . . . 161

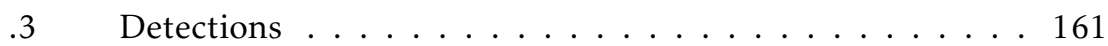

.4 Ground truth data . . . . . . . . . . . . . 162

False alarm reduction . . . . . . . . . . . . . . . 163

.1 Removing false detections using building segmentation . . 163

.2 Removing false detections using a classifier . . . . . . . 163

.3 Evaluation and selection methodology . . . . . . . . 165

Temporal analysis . . . . . . . . . . . . . . . . . . 167

Experimental results . . . . . . . . . . . . . . . 169

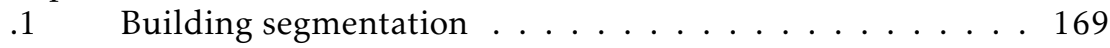

.2 Classification ....................... 169

.3 Summary of false alarm reduction results . . . . . . . 173

4 Temporal analysis . . . . . . . . . . . . . . 174

Conclusion ..................... 177

$\begin{array}{lr}\text { Bibliography } & 179\end{array}$ 
Part I

\section{Background Theory}





\section{1 \\ Introduction}

Thermal infrared cameras are becoming increasingly popular as prices and size decrease while image resolution and quality increase. An increasing number of people understand the potential and advantages of thermal infrared cameras. For example, their invariance to illumination changes and ability to see in total darkness. This thesis addresses the problems of detection and tracking specifically for thermal infrared imagery.

Five papers are included in the thesis. Paper A, B, and 7 are related to the field of tracking and Paper 8 and 9 describe detection methods for two applications. This introductory chapter provides a motivation for the thesis as well as describes its contributions and outline.

\subsection{Motivation}

Visual detection and tracking of objects in video are research areas that have been and currently are subject to extensive research. Approximately 40 motion or tracking papers are accepted in high profile conferences annually. Recent benchmarks indicate that they still remain challenging problems (Young and Ferryman (2005); Kristan et al. (2013, 2014, 2015, 2016); Wu et al. (2013, 2015); Li et al. (2016); Goyette et al. (2012); Wang et al. (2014b)). Both tracking and detection are relevant in many applications, mainly related to surveillance but also industry, scientific research (e.g. tracking animals in order to analyse behaviours), and automotive safety.

Detection and tracking in thermal infrared imagery has historically been of interest mainly for military purposes. Increasing image quality and resolution combined with decreasing price and size during recent years have, however, opened up new application areas. Thermal cameras are advantageous in many applications due to their ability to see in total darkness, their robustness to illumination 
changes, and less intrusion on privacy. Paper 8 and 9 address detection in thermal infrared and Paper 7 proposes a tracking method for thermal infrared imagery.

Common performance metrics and datasets are necessary for comparison of tracking results between different tracking methods. Without the existence of a common dataset that is sufficiently challenging, publications presenting new tracking methods tend to use proprietary datasets for evaluation. Consequently making it difficult to get an overview of the current status and advances within the field. Paper A argues that existing datasets for benchmarking of tracking methods in thermal infrared imagery have become outdated. The paper presents a new, publicly available, more challenging, thermal infrared benchmark for short-term single-object (STSO) tracking methods.

The results of the first ever organized challenge on STSO tracking for thermal infrared, VOT-TIR2015, are summarized in Paper B. The challenge had 24 participating trackers and was motivated by the lack of related work within the field of thermal infrared tracking benchmarks, only two previous occurrences were known.

Many applications connected to thermal cameras can be related to a sustainable society, for example, prevention and localisation of energy losses, as well as environmental friendly transportation. Two papers included in this thesis address these areas. Paper 9 addresses reduction of false alarms among automatically detected potential district heating leakages. Detection is performed in thermographic images captured with an airborne thermal camera. In addition, a method for temporal analysis of energy losses of a district heating network given two or more acquisitions of thermal imagery is presented. In Paper 8, an automatic method for rail and obstacle detection using a train-mounted thermal camera is presented. The system aims at providing an early warning to train drivers under impaired view. An early warning enables the driver to break before collision, significantly reducing repair costs.

\subsection{Contribution}

This thesis contains the following contributions:

- A dataset for evaluation of short-term tracking techniques: Paper A presents a publicly available dataset, LTIR (Linköping Thermal Infrared) ${ }^{1}$, of annotated thermal image sequences to be used for evaluation of short-term single-object tracking methods.

- The first ever organized challenge on short-term single-object tracking in thermal infrared imagery: The results of the VOT-TIR2015 challenge are presented in Paper B. It consists of an extensive benchmark of tracking performance of 24 participating short-term, single-object trackers.

- A short-term single-object tracking method designed for thermal infrared: Paper 7 describes a template-based tracking method, ABCD (Adaptive ob-

\footnotetext{
1 http://www.cvl.isy.liu.se/en/research/datasets/ltir/
} 
ject region and Background weighted scaled Channel coded Distribution field tracking), designed for thermal infrared.

- An anomaly based obstacle detection method based on adaptive correlation filters: A method for detecting obstacles on the railway is presented in Paper 8.

- Characterization and classification of automatically detected district heat leakages for false alarm reduction: Paper 9 applies learning methods to the problem of false alarm reduction among automatically detected district heating leakages. The paper also presents a method for temporal analysis of the status of a district heating network in the case of multiple acquisitions.

\subsection{Thesis Outline}

The thesis is divided into two parts. The rest of Part I presents the background theory for Part II, containing edited versions of published and submitted papers. Parts of the material presented in Part I has already been published by the author in technical reports and conference articles.

\subsubsection{Outline Part I: Background Theory}

Chapter 2 gives a brief overview of the physical principles related to thermal infrared imaging as well as explains its advantages and limitations. It also presents the main differences between image analysis in visual and thermal imagery and gives examples of applications. The contents of Chapter 2 are relevant for all included papers in this thesis.

Chapter 3, Detection and Tracking, is constituted of three sections, Section 3.2 Detection, Section 3.3 Tracking, and Section 3.4 Classification. The chapter begins with an overview, Section 3.1, where the three areas are related. The individual sections each introduces the subject as well as relates it to thermal infrared imagery. Background material for Paper A, B, and 7 is mainly presented in Section 3.3. Paper 8 is introduced in Section 3.2. Background theory for Paper 9 can be found in both Section 3.2 and 3.4. Finally, concluding remarks and future work are given in Chapter 4.

\subsubsection{Outline Part II: Included Publications}

Preprint versions of five papers are included in Part II. The full details and abstract of these papers, together with statements of the contributions made by the author, are summarized below.

\section{Paper A: A Thermal Object Tracking Benchmark}

A. Berg, J. Ahlberg, and M. Felsberg. A thermal object tracking benchmark. In Advanced Video and Signal Based Surveillance (AVSS), 2015 12th IEEE International Conference on, 2015a. 


\begin{abstract}
:
Short-term single-object (STSO) tracking in thermal images is a challenging problem relevant in a growing number of applications. In order to evaluate STSO tracking algorithms on visual imagery, there are de facto standard benchmarks. However, we argue that tracking in thermal imagery is different than in visual imagery, and that a separate benchmark is needed. The available thermal infrared datasets are few and the existing ones are not challenging for modern tracking algorithms. Therefore, we hereby propose a thermal infrared benchmark according to the Visual Visual Object Tracking (VOT) protocol for evaluation of STSO tracking methods. The benchmark includes the new LTIR dataset containing 20 thermal image sequences which have been collected from multiple sources and annotated in the format used in the VOT Challenge. In addition, we show that the ranking of different tracking principles differ between the visual and thermal benchmarks, confirming the need for the new benchmark.
\end{abstract}

\title{
Background and contribution:
}

This paper describes a new thermal infrared dataset (LTIR) for evaluation of short term, single object (STSO) trackers. Compared to previously available datasets, the LTIR dataset contains both 8 and 16 bit data, has higher resolution, more challenging sequences as well as sequences captured with both moving and stationary sensors. The LTIR dataset was also used in the first thermal infrared tracking challenge for STSO trackers, VOT-TIR2015 (Felsberg et al. (2015)). The author was part of developing the ideas for this paper, did the data collection and annotations, conducted experiments and did the main part of the writing.

\section{Paper B: The Thermal Infrared Visual Object Tracking VOT-TIR2015 Challenge Results}

M. Felsberg, A. Berg, G. Häger, J. Ahlberg, M. Kristan, J. Matas, A. Leonardis, L. Čehovin, G. Fernandez, and et al. The thermal infrared visual object tracking VOT-TIR2015 challenge results. In Computer Vision Workshops (ICCVW), IEEE International Conference on, pages 639651, Dec 2015. doi: 10.1109/ICCVW.2015.86.

\begin{abstract}
:
The Thermal Infrared Visual Object Tracking challenge 2015, VOT-TIR2015, aims at comparing short-term single-object visual trackers that work on thermal infrared (TIR) sequences and do not apply pre-learned models of object appearance. VOT-TIR2015 is the first benchmark on short-term tracking in TIR sequences. Results of 24 trackers are presented. For each participating tracker, a short description is provided in the appendix. The VOT-TIR2015 challenge is based on the VOT2013 challenge, but introduces the following novelties: (i) the newly collected LTIR (Linköping TIR) dataset is used, (ii) the VOT2013 attributes are adapted to TIR data, (iii) the evaluation is performed using insights gained during VOT2013 and VOT2014 and is similar to VOT2015.
\end{abstract}




\section{Background and contribution:}

In this paper, the results from the first ever organized challenge on short-term tracking in thermal infrared imagery are presented. The author was part of developing the ideas for the challenge, did the data collection and annotations, and did a main part of the writing. Note that the complete author list of the paper consists of 69 authors. According to the VOT-tradition, it is customary to include the authors of each participating tracker as an author of the result paper.

\section{Paper C: Channel Coded Distribution Field Tracking for Thermal Infrared Imagery}

A. Berg, J. Ahlberg, and M. Felsberg. Channel coded distribution field tracking for thermal infrared imagery. Submitted to IEEE PETS Workshop, 2016a

\section{Abstract:}

We address short-term, single-object tracking, a topic that is currently seeing fast progress for visual video, for the case of thermal infrared (TIR) imagery. The fast progress has been possible thanks to the development of new template-based tracking methods with online template updates, methods which have not been explored for TIR tracking. Instead, tracking methods used for TIR are often subject to a number of constraints, e.g., warm objects, low spatial resolution, and static camera. As TIR cameras become less noisy and get higher resolution these constraints are less relevant, and for emerging civilian applications, e.g., surveillance and automotive safety, new tracking methods are needed.

Due to the special characteristics of TIR imagery, we argue that templatebased trackers based on distribution fields should have an advantage over trackers based on spatial structure features. In this paper, we propose a template-based tracking method (ABCD) designed specifically for TIR and not being restricted by any of the constraints above. In order to avoid background contamination of the object template, we propose to exploit background information for the online template update and to adaptively select the object region used for tracking. Moreover, we propose a novel method for estimating object scale change. The proposed tracker is evaluated on the VOT-TIR2015 and VOT2015 datasets using the VOT evaluation toolkit and a comparison of relative ranking of all common participating trackers in the challenges is provided. Further, the proposed tracker, ABCD, and the VOT-TIR2015 winner SRDCFir are evaluated on maritime data. Experimental results show that the ABCD tracker performs particularly well on thermal infrared sequences.

\section{Background and contribution:}

In this paper, a template-based tracking method designed for thermal infrared imagery is presented. The method extends the EDFT (Felsberg (2013)) tracker to adaptively select the object region for tracking and to incorporate background information in the model update. The author developed the ideas for this paper, implemented the tracking method, conducted experiments and did the main part of the writing. 


\title{
Paper D: Detecting Rails and Obstacles using a Train-Mounted Thermal Camera
}

\begin{abstract}
A. Berg, K. Öfjäll, J. Ahlberg, and M. Felsberg. Detecting rails and obstacles using a train-mounted thermal camera. In Image Analysis, volume 9127 of Lecture Notes in Computer Science, pages 492-503. Springer International Publishing, 2015c.
\end{abstract}

\begin{abstract}
:
We propose a method for detecting obstacles on the railway in front of a moving train using a monocular thermal camera. The problem is motivated by the large number of collisions between trains and various obstacles, resulting in reduced safety and high costs. The proposed method includes a novel way of detecting the rails in the imagery, as well as a way to detect anomalies on the railway. While the problem at a first glance looks similar to road and lane detection, which in the past has been a popular research topic, a closer look reveals that the problem at hand is previously unaddressed. As a consequence, relevant datasets are missing as well, and thus our contribution is two-fold: We propose an approach to the novel problem of obstacle detection on railways and we describe the acquisition of a novel data set.
\end{abstract}

\section{Background and contribution:}

This paper describes new methods for rail detection and correction in thermal infrared imagery as well as anomaly detection of obstacles on the railway. The author was part of developing the ideas for this paper, implemented the anomaly detector and rail corrector, conducted experiments on the same, wrote Section 3 and 4 and was the main author of the paper.

\section{Paper E: Enhanced Analysis of Thermographic Images for Monitoring of District Heat Pipe Networks}

A. Berg, J. Ahlberg, and M. Felsberg. Enhanced analysis of thermographic images for monitoring of district heat pipe networks. Submitted to Pattern Recognition Letters (PRL), 2016b

\begin{abstract}
:
We address two problems related to large-scale aerial monitoring of district heating networks. First, we propose a classification scheme to reduce the number of false alarms among automatically detected leakages in district heating networks. The leakages are detected in images captured by an airborne thermal camera, and each detection corresponds to an image region with abnormally high temperature. This approach yields a significant number of false positives, and we propose to reduce this number in two steps; by (a) using a building segmentation scheme in order to remove detections on buildings, and (b) to use a machine learning approach to classify the remaining detections as true or false leakages. We provide extensive experimental analysis on real-world data, showing that this post-processing step significantly improves the usefulness of the system. Second,
\end{abstract}


we propose a method for characterization of leakages over time, i.e., repeating the image acquisition one or a few years later and indicate areas that suffer from an increased energy loss. We address the problem of finding trends in the degradation of pipe networks in order to plan for long-term maintenance, and propose a visualization scheme exploiting the consecutive data collections.

\section{Background and contribution:}

In this journal article, methods for large-scale monitoring of district heating networks are described. The article focuses on the reduction of false alarms among automatically detected areas with abnormally high temperatures. In addition, a method and visualization technique for temporal analysis given several acquisitions of the same area are proposed. The author was part of developing the ideas for this paper, did the data collection and annotations, conducted experiments and did the main part of the writing.

\subsection{Other Publications}

The following publications by the author are related to the included papers.

A. Berg, J. Ahlberg, and M. Felsberg. A thermal infrared dataset for evaluation of short-term tracking methods. In Swedish Symposium on Image Analysis (SSBA), 2015b. (Early version of Paper A)

A. Berg, M. Felsberg, G. Häger, and J. Ahlberg. An overview of the thermal infrared visual object tracking VOT-TIR2015 challenge. In Swedish Symposium on Image Analysis (SSBA), 2016c. (Overview of Paper B)

A. Berg and J. Ahlberg. Classification of leakage detections acquired by airborne thermography of district heating networks. In Pattern Recognition in Remote Sensing (PRRS), 2014 8th IAPR Workshop on, 2014b. (Early version of Paper 9)

A. Berg and J. Ahlberg. Classification and temporal analysis of district heating leakages in thermal images. In The 14th International Symposium on District Heating and Cooling (DHC), 2014a. (Early version of Paper 9)

A. Berg, J. Ahlberg, and M. Felsberg. Classifying district heating network leakages in aerial thermal imagery. In Swedish Symposium on Image Analysis (SSBA), 2014. (Very early version of Paper 9)

J. Ahlberg and A. Berg. Evaluating template rescaling in short-term single-object tracking. In Advanced Video and Signal Based Surveillance (AVSS), 2015 12th IEEE International Conference on, pages 1-4, Aug 2015. doi: 10.1109/AVSS.2015.7301745. 


\subsection{Figures}

Previously published figures and tables are reproduced with permission of the respective copyright holder. For unlisted figures, either the copyright remains with the author (SSBA) or the figures are made specifically for this publication.

\subsubsection{Springer}

The copyright of the figures and tables from the following publications resides with Springer and are reproduced with permission of Springer.

A. Berg, K. Öfjäll, J. Ahlberg, and M. Felsberg. Detecting rails and obstacles using a train-mounted thermal camera. In Image Analysis, volume 9127 of Lecture Notes in Computer Science, pages 492-503. Springer International Publishing, 2015c

\subsubsection{IEEE}

The copyright of the figures and tables from the following publications resides with IEEE and are reproduced with permission of IEEE.

A. Berg, J. Ahlberg, and M. Felsberg. A thermal object tracking benchmark. In Advanced Video and Signal Based Surveillance (AVSS), 2015 12th IEEE International Conference on, 2015a

M. Felsberg, A. Berg, G. Häger, J. Ahlberg, M. Kristan, J. Matas, A. Leonardis, L. Čehovin, G. Fernandez, and et al. The thermal infrared visual object tracking VOT-TIR2015 challenge results. In Computer Vision Workshops (ICCVW), IEEE International Conference on, pages 639651, Dec 2015. doi: 10.1109/ICCVW.2015.86 


\section{2}

\section{Thermal Infrared Imaging}

Thermal infrared imaging forms the basis of this thesis. All included papers apply automatic image analysis to thermal images. The following chapter gives a brief overview of the physics behind thermal infrared imaging as well as image analysis in thermal infrared. It also explains the relation between infrared light and the planet Uranus, shows a wooden rhino in different color maps, and explains how thermal cameras can be used for bat research. The chapter concludes with some application examples.

\subsection{Infrared and thermal radiation}

Infrared radiation was originally discovered in 1800 by Sir Frederick William Herschel (1738-1822), who is also famous for discovering the planet Uranus as well as writing 24 symphonies (Rogalski (2012)). Infrared radiation is a part of the electromagnetic spectrum, see Fig. 2.1, and its name originates from the Latin word infra, which means below. That is, the infrared band lies below the visual red light band, since it has longer wavelength.

The infrared wavelength band is broad and is usually divided into different bands based on their different properties: near infrared (NIR, wavelengths 0.7$1 \mu \mathrm{m}$ ), shortwave infrared (SWIR, 1-3 $\mu \mathrm{m}$ ), midwave infrared (MWIR, 3-5 $\mu \mathrm{m}$ ), and longwave infrared (LWIR, 8-12 $\mu \mathrm{m}$ ). Other definitions exist as well. LWIR, and sometimes MWIR, is commonly referred to as thermal infrared (TIR). TIR cameras are sensitive to emitted radiation in everyday temperatures and should not be confused with NIR and SWIR cameras that, in contrast, mostly measure reflected radiation. These non-thermal cameras are dependent on illumination and behave in general in a similar way as visual cameras.

When interacting with matter, electromagnetic radiation is absorbed $(\alpha)$, transmitted $(\tau)$ and/or reflected $(\rho)$. The total radiation law states that $1=\alpha+\rho+\tau$ 


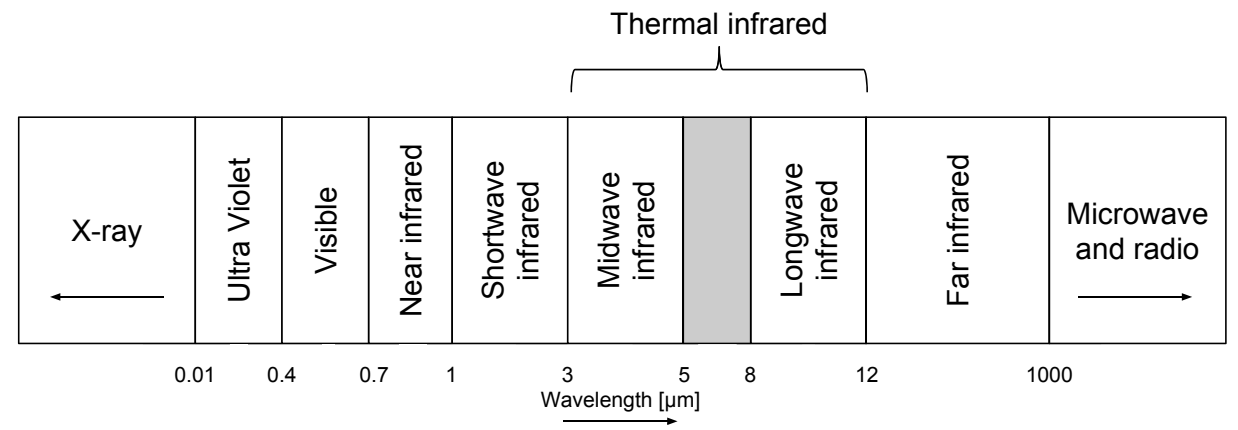

Figure 2.1: Infrared radiation is a part of the electromagnetic spectrum. Longwave, and sometimes midwave, infrared is commonly referred to as thermal infrared. Between 5-8 $\mu \mathrm{m}$, the atmosphere attenuates most of the radiation, see Fig. 2.4 .

where $\alpha, \tau, \rho \in[0,1]$. In addition, an object's thermal energy can be converted into electromagnetic energy, called thermal radiation. All objects with temperatures above absolute zero emit thermal radiation to a different extent depending on temperature and material.

An object defined as a black body is an opaque and non-reflective object that absorbs all incident radiation $(\alpha=1)$. Black bodies do not exist in nature, but are commonly used as an approximation. Examples of black body radiation curves for some known objects can be seen in Fig. 2.2. Note that the peak of the sun lies in the reflective part of the electromagnetic spectrum.

Emissivity $(\epsilon)$ is the ratio of the actual emittance of an object to the emittance of a black body at the same temperature. Further, Kirchhoff's law states that $\alpha=\epsilon$, i.e., $\epsilon=1$ for a black body. Since emissivity is material dependant, it is an important property when measuring temperatures with a thermal camera. An example of how the emissivity of an object can affect what is perceived is given in Fig. 2.3.

Due to scattering by particles and absorption by gases, the atmosphere will attenuate radiation, making the measured apparent temperature decrease with increased distance. The level of attenuation depends on radiation wavelength, Fig. 2.4. As can be seen in the figure, there are two main sections in which the atmosphere transmits a major part of the radiation. These are called the atmospheric windows and can be found between 3-5 $\mu \mathrm{m}$ (the mid-wave window) and 8-12 $\mu \mathrm{m}$ (the long-wave window) (Rees (2001)). These windows correspond to the MWIR and LWIR bands mentioned above.

This section only provides a brief overview of the topic, for more information on thermal infrared detectors and physical principles, see e.g. (Rees (2001); Rogalski (2012)). 


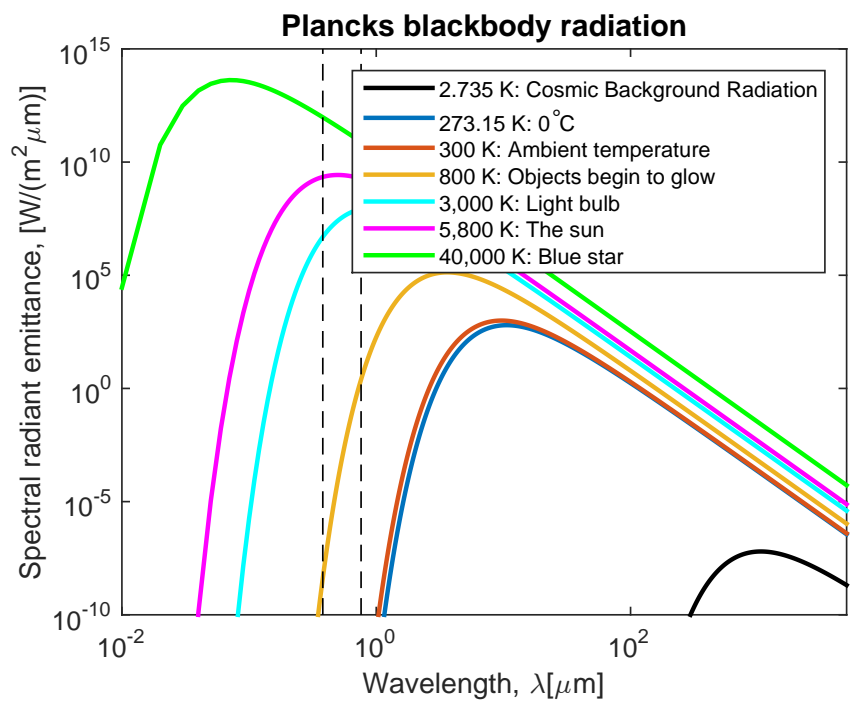

Figure 2.2: Black body radiation for different objects. As the temperature increases, the peak of the emitted radiation moves towards shorter wavelengths and higher intensities. The dashed lines marks the visual part of the electromagnetic spectrum.

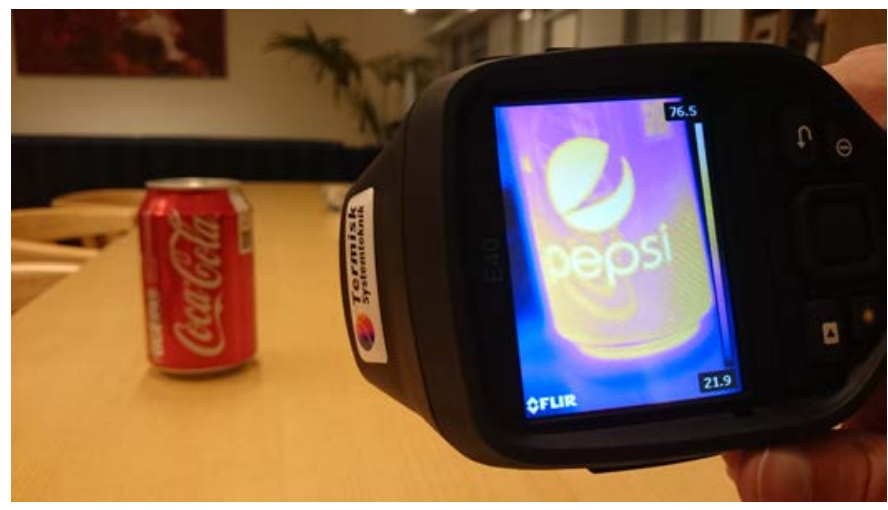

Figure 2.3: An example of how the emissivity of materials affects what is perceived. A transparent tape of another logo than that of the soda has been placed on the metal can. The can was then filled with hot water. The tape has higher emissivity than the can and appears warmer when measuring. Image courtesy of Jörgen Ahlberg and Patrik Stensbo. 


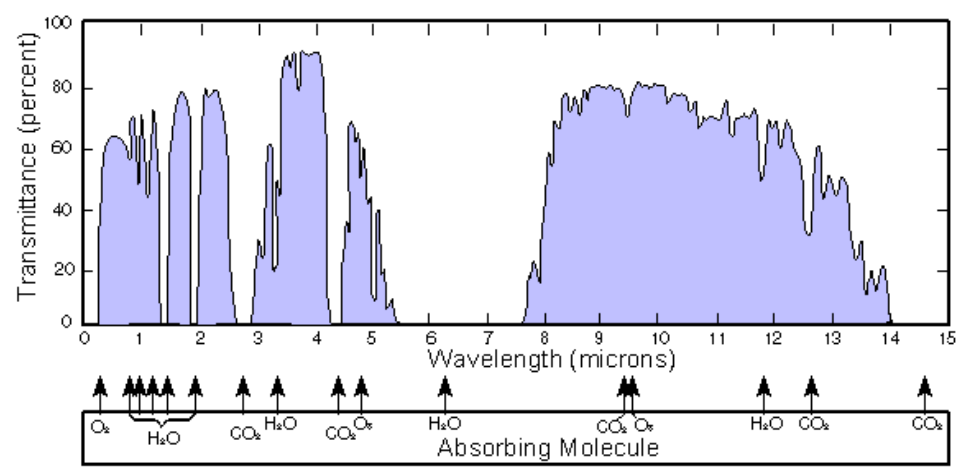

Figure 2.4: Atmospheric attenuation depends on radiation wavelength, therefore, thermographic measurements are done within one of the two atmospheric windows. The midwave window, 3-5 $\mu \mathrm{m}$, or the longwave window 8-12 $\mu \mathrm{m}^{1}$.

\subsection{Thermal imaging}

Thermal images are visual displays of measured emitted, reflected, and transmitted thermal radiation within an area. When presented to an operator, color maps are often used to map pixel intensity values in order to visualize details more clearly. Examples of a thermal image and widely used color maps can be seen in Fig. 2.5.

Due to multiple sources of thermal radiation, thermal imaging can be challenging depending on the properties of the object and its surroundings, see Fig. 2.6. The amount of radiation emitted by the object depends on its emissivity as explained in the previous section. In addition, thermal radiation from other objects are reflected on the surface of the object. Therefore, it is also important to know the reflectivity of the object. The amount of radiation that reaches the detector is affected by the atmosphere. Some is transmitted, some is absorbed, and some is even emitted from the atmosphere itself. Moreover, the camera itself emits thermal radiation during operation. In order to measure thermal radiation and thus temperatures as accurately as possible, all these effects need to be considered. At short distances, atmospheric effects can be disregarded. But for greater distances, e.g. from aircrafts as in Paper 9, it is crucial to consider atmospheric effects if temperatures are to be measured correctly. However, if you are only interested in an image that looks good to the eye and not temperatures, these effects do not have to be taken into account.

Materials have different properties in the thermal spectrum compared to the visual. Some materials that are reflective and/or transparent in the visual spectrum are not in the thermal spectrum and vice versa, e.g., glass. Glass is transpar-

\footnotetext{
${ }^{1}$ Source: https://en.wikipedia.org/wiki/File:Atmosfaerisk_spredning.gif Accessed: 2016-01-04
} 


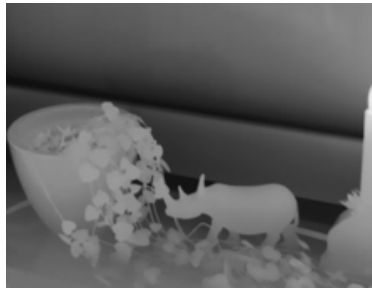

(a) Gray

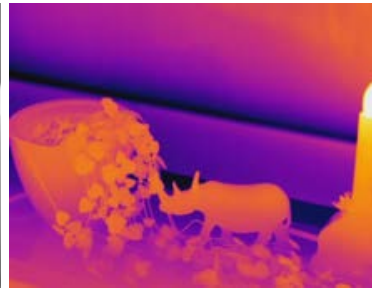

(b) Iron

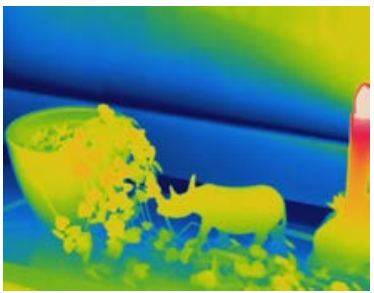

(c) Rainbow

Figure 2.5: Example of a thermal image visualized using different color maps.

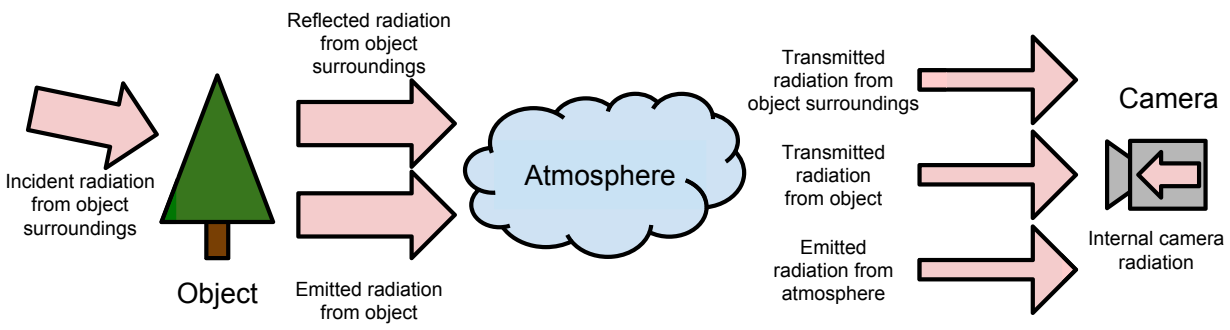

Figure 2.6: Influences on what the thermal camera measures.

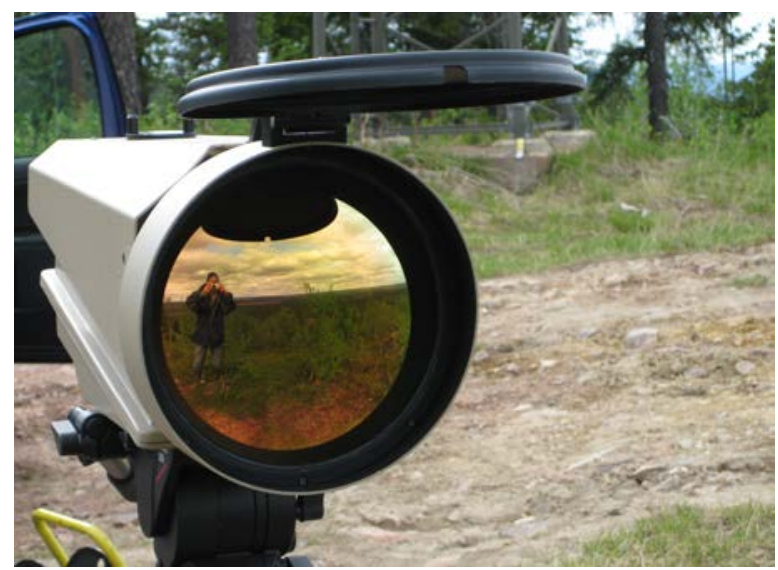

Figure 2.7: Example of a germanium lens. Germanium is opaque and reflective in the visual spectrum while transparent in the thermal spectrum. Image courtesy of Magnus Uppsäll. 
ent in the visual spectrum while being opaque in the thermal spectrum, therefore, the lens of a thermal camera is not manufactured in glass but in germanium, a material that is opaque and reflective in the visual spectrum but transparent in the thermal spectrum, Fig. 2.7.

Thermal cameras are either cooled or uncooled. High-end cooled cameras can deliver hundreds of HD resolution frames per second and have a temperature sensitivity of $20 \mathrm{mK}$. Images are typically stored as 16 bits per pixel to allow a large dynamic range, for example $0-382.2 \mathrm{~K}$ with a precision of $10 \mathrm{mK}$. Uncooled cameras usually have bolometer detectors and operate in LWIR. They yield noisier images at a lower framerate, but are smaller, silent, and less expensive.

A thermal camera is said to be thermographic if it is calibrated in order to measure temperatures. Some uncooled cameras provide access to the raw 16-bit intensity values, so called radiometric cameras, while others convert the images to 8 bits and compress them e.g. using MPEG. In the latter case, the dynamic range is adaptively changed in order provide an image that looks good to the eye, but the temperature information is lost. For automatic analysis, such as target detection, classification, and tracking, it is suitable to use the original signal, i.e., the raw 16-bit intensity values from a radiometric camera.

\subsection{Advantages and limitations of thermal imaging}

From the aspect of measuring temperatures, thermal imaging is advantageous compared to point-based methods since temperatures over a large area can be compared. However, it is not considered to be as accurate as contact methods.

Compared to visual cameras, thermal cameras are favourable as soon as there is a temperature difference connected to the object or phenomena you want to detect. For example, emerging fires, humans, animals, increased body temperatures, or differences in heat transfer ability in materials. When it comes to applications, thermal cameras are especially advantageous to visual cameras in outdoor applications. Thermal cameras can produce an image with no or few distortions during darkness and/or difficult weather conditions (e.g. fog/rain/snow). This is again due to the fact that a thermal camera is sensitive for emitted radiation, even from relatively cold objects, in contrast to a visual camera that measures reflected radiation and thus depends on illumination.

Thermal cameras are expensive and have low resolution compared to visual cameras. State of the art is currently $1344 \times 784$ pixels, and increased resolution comes with a higher price tag, up to $€ 200000$. Prices depend on the choice of detector (cooled/uncooled, MWIR/LWIR), optics etc.

In comparison to a visual camera, a thermal camera typically requires more training for correct usage. In order to provide accurate measurements, the operator needs to be aware of the physical principles and phenomena commonly viewed in thermal imagery. That is, the emissivity and reflectivity of different materials as well as the impact of the atmosphere, Fig. 2.6.

From thermal imagery, it is not considered possible to perform person identification, a fact that is both an advantage as well as a limitation. It means that a 
thermal camera can be used in applications where preservation of privacy is crucial. However, if person identification is requested, it has to be combined with a visual camera.

\subsection{Image analysis in thermal infrared}

In this section, differences between thermal and visual imagery when performing automatic image analysis are described. Some descriptions are intentionally left brief since they are further described in Sections 3.2 and 3.3 in relation to detection and tracking in thermal infrared.

First, as mentioned in Section 2.2, materials have different properties in the thermal and visual spectrum respectively. For example, water and glass are opaque and highly reflective in the thermal spectrum while plastic bags are not. Glass, water puddles and wet soil can cause reflections similar to shadows. In the thermal infrared spectrum, there are no shadows since mostly emitted radiation is measured. In most applications, the emitted radiation changes much slower than the reflected radiation. That is, an object moving from a dark room into the sunlight will not immediately change its appearance (as it would in visual imagery).

Regarding noise, thermal imagery has different characteristics than visual imagery. Compared to a visual camera, a thermal infrared camera typically has more blooming, lower resolution and a larger percentage of dead pixels. Visual color patterns are discernible in thermal infrared images only if they correspond to variations in material or temperature.

Finally, a thermal infrared camera is itself a source of thermal radiation. During operation, especially during start-up, it heats itself. The radiation reaching the sensor can to a large part originate from the camera itself. To compensate for this, thermal infrared cameras typically have internal thermometers and they also perform radiometric calibration at regular time intervals. During calibration, a plate with known temperature is inserted in front of the sensor, and frames are lost.

\subsection{Applications}

There are many applications related to automatic image analysis in thermal imagery. As image resolution and quality increase and prices decrease there will be even more, not even thought of yet. A few categories have been identified and described below and example images from each category can be seen in Fig. 2.8.

Agriculture: Monitoring of wild and domestic animals can be used, e.g., to detect inflammations, perform behaviour analysis, or to estimate population sizes (Hristov et al. (2010); Gan et al. (2015); Colak et al. (2008)).

Automotive safety: Detection and tracking of pedestrians, but also other vehicles, using a small thermal camera mounted in the front of a car (or train) (Berg 
et al. (2015c); Bertozzi et al. (2004); Zhang et al. (2007)).

Building inspection: Heat losses in buildings can be detected using a thermal camera. There are automatic methods that maps thermal images to 3D models for heat loss visualization (Hoegner and Stilla (2009)).

Fire monitoring: Thermal (radiometric) cameras are useful for detecting fires. They can also see through smoke and are commonly used by fire fighters to find persons and to localise the base of the fire (Paugam et al. (2013); Rydell and Bilock (2015)).

Industry: Industry is a broad area that has many applications. Detection of different materials, positioning, non-destructive testing etc. (Berg et al. (2016b); Runnemalm et al. (2014); Ng et al. (2007)).

Medical: Detection of tumours in early stages, inflammations, fever screening, etc. (Ahlberg et al. (2015); Chekmenev et al. (2007); Lahiri et al. (2012)).

Military: There are numerous military applications, such as target tracking, gunfire detection, missile approach warning, mine detection, and sniper detection (Siegel (2002)). Military applications will not be treated in this thesis.

Personal use: Recently, a small thermal camera that can be attached to a smart phone was released. This is likely to spawn new applications not yet imagined. For example in sports where a thermal camera can be used to obtain a visual image of the heat in your muscles.

Search and rescue: Searching for persons independently of daylight using cameras carried by UAVs, helicopters or rescue robots (Rudol and Doherty (2008)).

Security: Detection, tracking, and behaviour analysis of persons and vehicles for detection of intrusion and suspicious behaviour (Berg et al. (2015a); Davis and Sharma (2004); Han and Bhanu (2005)). 


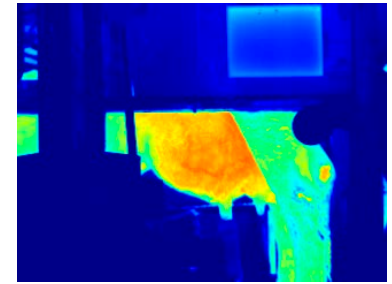

(a) Agriculture

Detection of mastitis ${ }^{\dagger}$.

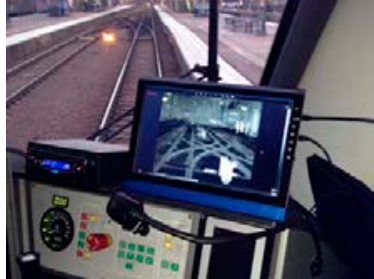

(b) Automotive safety

Obstacle detection in front of trains ${ }^{\star}$. With permission of Springer.

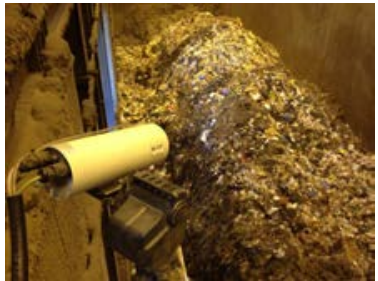

(d) Fire monitoring Monitoring flammable waste ${ }^{\star}$.

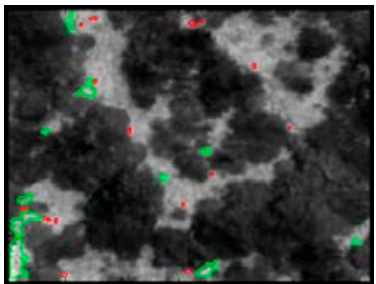

(g) Military

Mine detection (Linderhed et al. (2004)).

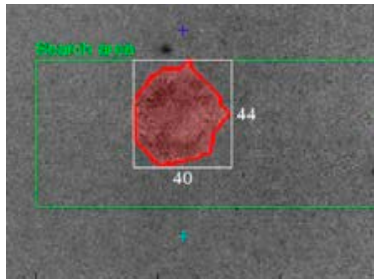

(e) Industry

of Inspection of spot welds ${ }^{\star}$.

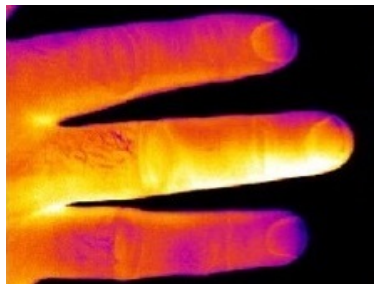

(h) Personal use Checking infections ${ }^{\ddagger}$.

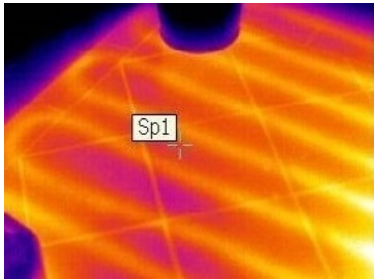

(c) Building inspection Inspection of floor heating ${ }^{\ddagger}$.

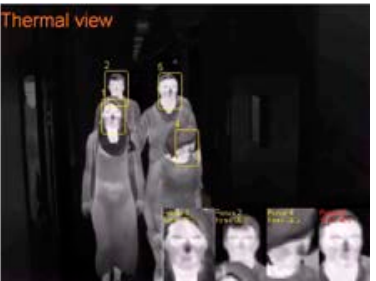

(f) Medical

Fever screening of crowds $s^{\diamond}$

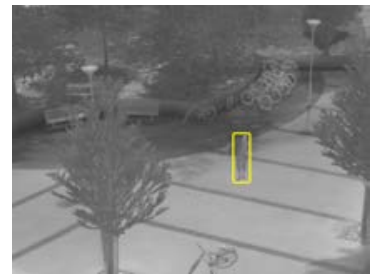

(j) Security Intrusion detection ${ }^{\star}$

Figure 2.8: Thermal imagery application examples. Image courtesy of $\star$ Termisk Systemteknik $A B,{ }^{\dagger}$ Agricam $A B, \ddagger$ FLIR $A B$, and ${ }^{\diamond}$ Imafor $A B$. 



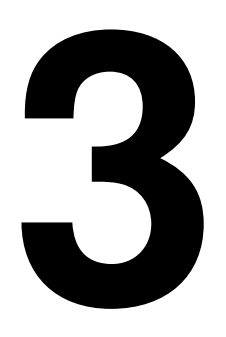

\section{Detection and Tracking}

This chapter introduces detection and tracking and provides more details on the specific challenges of detection and tracking in thermal infrared imagery. In addition, the field of classification is briefly described.

Section 3.1 describes the relation between detection, tracking and classification while Section 3.2, 3.3, and 3.4, address each area in more detail. Background material for Papers A, B, and 7 is mainly presented in Section 3.3. Paper 8 is introduced in Section 3.2. Background theory for Paper 9 can be found in both Section 3.2 and 3.4.

\subsection{Relation between detection, tracking, and classification}

Detection and tracking are two closely related problems. Tracking is dependant on accurate detection of the objects to be tracked. Such objects can be anomalous or pre-learnt. Imagine a high-dimensional representation that has been used to create a model of a background. If the background model is adaptive, the model is allowed to change over time as it adapts to gradual changes of the environment. The background model can also be learnt. The difference between adaptive and learnt models is that a learnt model can recognize phenomena it has seen before. An adaptive model has a shorter memory span but can adapt to previously unseen changes. Fig. 3.1a illustrates a background model. Samples of background have been used to create the model in this representation, and a decision line has been formed around the model. The decision line can, e.g., be a distance measurement from a distribution or formed using a hyperplane whose parameters have been learnt from examples. Samples outside this line are not considered to be background. 


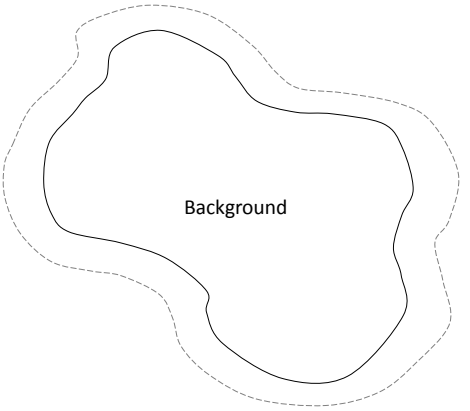

(a) Background model

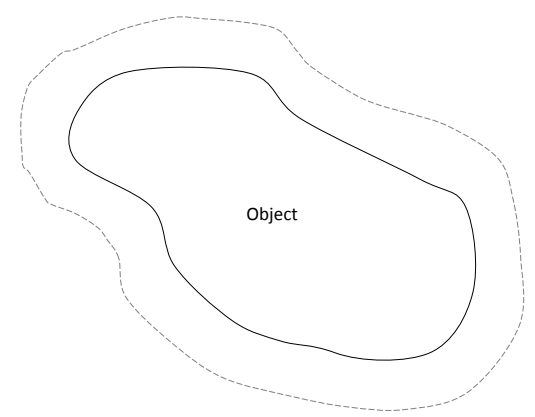

(c) Template-based tracking, detection of specific objects

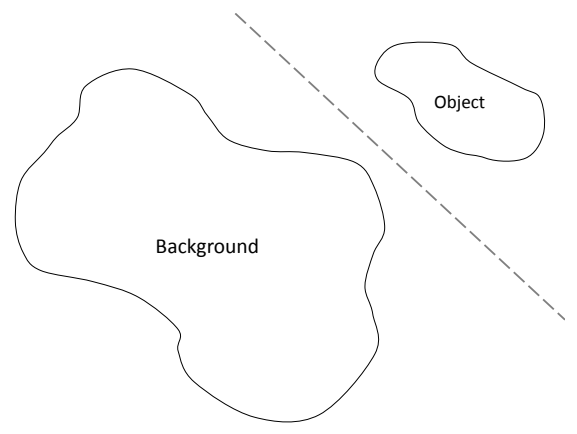

(e) Template-based tracking

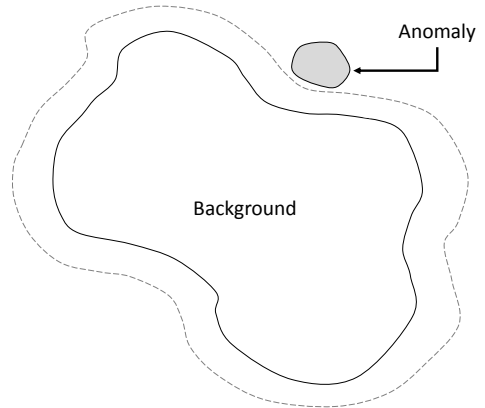

(b) Anomaly detection

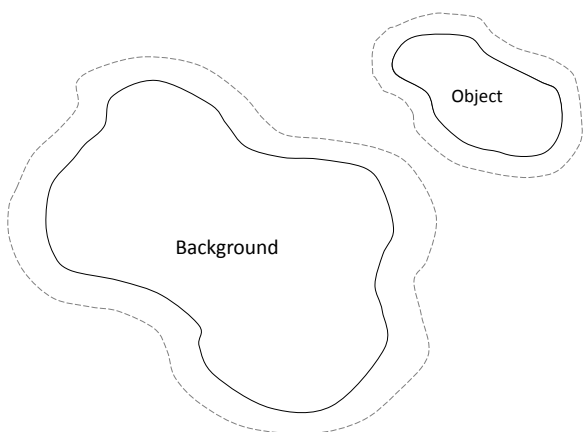

(d) Template-based tracking

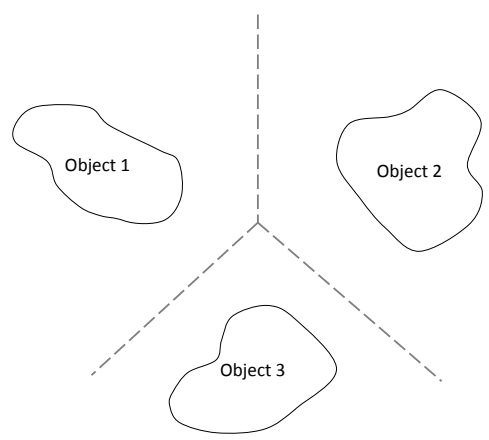

(f) Classification

Figure 3.1: A visual illustration of the relation between detection, tracking, and classification. Dashed lines represent decision boundaries. 
Samples appearing outside the decision line, Fig. 3.1b, are regarded as anomalies. Detection of anomalies, Section 3.2.1, can be defined as detection of samples outside a decision line. The decision line does not have to be fixed, it can be adaptively updated in order to agree with a continuously updated background model. Decisions can also be taken using multiple decision lines, so called hysteresis.

In the case of template-based tracking, Section 3.3.2, a model of an object is created and continuously updated. A decision line is formed around the object in order to correctly match the object model to a tentative object patch, see Fig. 3.1c. Detection of specific objects, Section 3.2.2, can be described with the same figure. There are template-based methods, for example the one presented in Paper 7, that, in addition, creates a model of the background in order to make a more accurate distinction of background and object and to prevent background contamination of the object model, Fig. 3.1d and 3.1e.

Finally, classification, which is the assignment of samples to different class labels, basically implies forming decision lines between all learnt object models, Fig. 3.1f and Section 3.4.

The above text illustrates the similarities and differences of detection, tracking, and classification. The subsequent sections contain a more detailed description of the different areas. Detection and tracking specifically for thermal infrared imagery are treated in Section 3.2.3 and 3.3.5.

\subsection{Detection}

In our context, detection is the technique of finding specific or anomalous objects in images or video. In this section, detection techniques are introduced and detection specifically for thermal infrared imagery is described.

\subsubsection{Anomaly detection}

When humans apply anomaly detection to video, we tend to use pre-learnt knowledge about what constitutes the normality, i.e., the background in the scene. For example, imagine a typical surveillance video with a tree swaying slightly in the wind. A human would probably not consider that to be abnormal since it is just a tree. For a computer vision algorithm, it might be more difficult to separate the motions from a tree from the motions of a person doing reconnaissance.

Here, two examples of anomaly detection techniques are given: background subtraction and adaptive correlation filtering.

\section{Background subtraction}

Background subtraction, also known as change detection, is one possible way of performing anomaly detection. It is the technique of learning a background model and classifying all samples not belonging to the background as foreground, or anomalies. The greatest challenge when it comes to background subtraction 
is probably the gray areas when deciding whether a region belongs to the foreground or not. For example, a background object can be moved while still being a background object. Another possible scenario is someone starting a parked car and driving away; the car should not be considered a background object anymore. What moved background objects are still to be considered as background and which are not? Described below are challenges connected to background subtraction identified by Toyama et al. (1999), and how they relate to thermal imagery:

Bootstrapping: Background subtraction methods often require a training period absent of foreground objects. Such a period is not available in all applications.

Camouflage: In visual as well as thermal imagery, there may be objects that, intentionally or not, appear similar to the background. Further, visual colour patterns are only discernible in thermal infrared if they correspond to differences in temperature.

Foreground aperture: The problem of detecting changes in object interior pixels in the case of homogeneously coloured objects is known as the foreground aperture problem. This problem is more common in thermal infrared due to a different noise characteristics with more blur and less discernible patterns. Thus, objects with a homogeneous intensity might not be detected completely.

Light switch: Sudden illumination changes that alter the appearance of the background can cause false detections. This phenomenon does not exist in thermal to the same extent as is visual imagery since emitted radiation change much slower than reflected.

Moved objects: If a background object is moved, it should not be considered foreground forever after.

Shadows: Shadows cast by foreground objects can complicate processing steps subsequent to background subtraction. The shadows can themselves be detected as foreground objects, or, overlapping shadows of foreground objects can, for example, hinder the separation and classification of the objects. Shadows do not exist in thermal infrared imagery, but areas visually similar to shadows may appear for stationary objects. Shaded areas in the visual domain will not be as heated as areas that are not. This implies that objects that cast shadows that have been standing still and start moving will leave what looks like a shaded area behind, since that area has not been as heated as its surroundings.

Sleeping person: A foreground object that becomes motionless, e.g., a person falling asleep, can not be distinguished from a moving background object that becomes motionless.

Time of day (gradual illumination/dynamics changes): Gradual illumination 
changes affect the appearance of the background, this is especially problematic in outdoor applications. There are less illumination changes in thermal infrared since mostly emitted and not reflected radiation is measured. However, the amount of emitted radiation can also vary but it varies much slower that reflected radiation. For example, consider a person walking from shadow into the sun. The lightning of the person will immediately change but the person will not immediately change temperature. Furthermore, due to the fact that many thermal sensors do not allow access to the raw, 16-bit, data, the data is commonly truncated to 8 -bits with a variable range which creates dynamics changes. A background model should be able to adapt to these gradual changes of the appearance of the environment.

Walking person: If a motionless object, initially part of the background, starts moving. Then both the object and the newly revealed parts of the background will be considered as foreground.

Waving trees/dynamic background: There may be parts of the scenery that contain movements that should be regarded as background. There could, for example, be traffic lights (periodical) and grass swaying in the wind (irregular). Dynamic backgrounds require representations that can model a disjoint set of features. Dynamic backgrounds exist in both visual and thermal infrared.

There are some additional challenges which are not identified by Toyama et al. (1999):

Moving sensor: A moving sensor will cause the background to appear as if it is moving.

Reflections: Reflections of objects in highly reflective surfaces can cause problems similar to those of shadows.

Video noise/degraded signals: A video signal is generally degraded by noise to different degrees. There are different types of noise, e.g., sensor noise or compression artefacts. In thermal infrared, the noise characteristics is slightly different compared to visual cameras, a thermal camera typically has lower resolution, more blooming, and a larger percentage of dead pixels. In addition, radiometric calibration causes frames to be lost at regular time intervals, see Section 2.4.

Background subtraction is a broad research topic and a complete review of techniques is out of scope for this thesis. An indication of its popularity are the Change Detection workshops (Goyette et al. (2012); Wang et al. (2014b)) and website $^{1}$ that offer datasets and benchmarks for performance evaluation of change detection techniques. The benchmarks even host a thermal infrared subcategory.

\footnotetext{
$1_{\text {http: } / / \text { ww }}$.changedetection. net
} 
The top performing background subtraction techniques for thermal infrared imagery at the time of writing are FTSG (Flux Tensor with Split Gaussian models) (Wang et al. (2014a)), CwisarDH (Gregorio and Giordano (2014)), and Spectral360 (Sedky et al. (2014)) all of which are top-performing methods also in the overall category.

\section{Adaptive correlation filters}

Adaptive/discriminative correlation filters (DCF's) can be used for a number of different applications. Examples within the research area of computer vision are, visual tracking (Danelljan et al. (2014); Henriques et al. (2015)), object detection (Galoogahi et al. (2013); Henriques et al. (2013)), and object alignment (Boddeti et al. (2013)).

The object detection methods referenced above (Galoogahi et al. (2013); Henriques et al. (2013)) address detection of specific objects, e.g. pedestrians. DCF's can, however, also be used for anomaly detection in the case of repetitive background. That is, an anomaly is defined as a failure to detect background.

A DCF based approach models the appearance of the object by adaptive, discriminative correlation filters and detection is performed via convolution. The MOSSE tracker (Bolme et al. (2010)) introduced a regularized adaptive correlation filter suitable for visual tracking. Danelljan et al. (2014) extended the work of Bolme et al. (2010) and combined a two-dimensional filter for tracking and a one-dimensional filter for scale estimation.

The Convolution Theorem states that correlation becomes an element-wise multiplication in the Fourier domain. Therefore, all filter operations are performed in the Fourier domain using the Fast Fourier Transform in order to reduce computation time. Correlation in the Fourier domain between a filter $H$ and an image patch $F$ takes the form:

$$
G=F \odot \bar{H} .
$$

The bar denotes complex conjugation and $\odot$ represents element-wise multiplication. DCF based approaches find a filter $H$ that minimizes the sum of squared errors of the difference between the desired output $G_{j}$ and the convolution $F_{j} \odot \bar{H}$ for a number of training samples $j=1, \ldots, J$, i.e.

$$
\min _{\bar{H}} \sum_{j}\left|F_{j} \odot \bar{H}-G_{j}\right|^{2} .
$$

The desired output, $G_{j}$ is the Fourier transform of the ideal filter response, typically a Gaussian with its peak centred at the target. The solution to (3.2) is:

$$
\bar{H}=\frac{\sum_{j} G_{j} \odot \overline{F_{j}}}{\sum_{j} F_{j} \odot \overline{F_{j}}},
$$

where division is performed element-wise. A complete derivation is given in Bolme et al. (2010). The predicted position of the detected object at time $t$ is found at the maximum value of the filter response $y_{t}$ : 


$$
y_{t}=\mathcal{F}^{-1}\left\{\overline{H_{t}} Z\right\},
$$

where $Z$ is an image patch of a search area. If new examples of the object are provided and added to the set of training samples, an optimal filter can be obtained by minimizing the output error over the complete set. This approach is, however, not feasible for online learning applications. Instead, the filter is adaptively updated using a weighted average with update factor $\alpha \in[0,1]$ as

$$
H_{t}=\frac{A_{t}}{B_{t}},
$$

where

$$
\begin{aligned}
& A_{t}=(1-\alpha) A_{t-1}+\alpha\left(\bar{G} \odot F_{j}\right), \\
& B_{t}=(1-\alpha) B_{t-1}+\alpha\left(\overline{F_{j}} \odot F_{j}\right) .
\end{aligned}
$$

In order to reduce the problem of zero-frequency components in $F$ leading to division by zero, a regularization parameter $\lambda$ can be added to (3.2), see Bolme et al. (2010).

Paper 8 utilizes a one-dimensional DCF for anomaly detection. The paper addresses the problem of obstacle detection in front of a train when using thermal imagery from a train-mounted camera. Here, due to the known geometry and repetitive nature of the area between rails, a one-dimensional, horizontal filter is employed. The filter is applied row-wise within a rail mask provided by a preceding rail detection step.

\subsubsection{Detection of specific objects}

The term specific objects can have different meanings depending on the level of specificity. It can be a specific class of objects, humans, all humans with blue jackets, or even a specific human. Depending on the level of specificity the detection approach can be slightly different. When detecting a specific class of objects, the important part for the method is the ability to generalize. What are the common attributes for all humans? In order to do this, a large number of training samples are typically needed. Detecting a specific object from frame to frame is treated in Section 3.3.2 on template-based tracking.

When detecting specific objects, the first step is to determine ROI's (regions of interest) in order to produce a number of initial hypotheses. The most common method is the sliding window approach, which can be an exhaustive search method. It shifts windows of different scales and positions across the image. Due to its computational complexity it is, however, most often optimized in order to allow for real-time processing. Prior knowledge about the object (size, previous motion etc.) can be used to limit the search space, and/or a classifier cascade with increasing complexity (Markuš et al. (2014)). Another common method is to use background subtraction, described more thoroughly above.

ROI selection gives us a number of initial hypotheses that are commonly used as input to a learning method. The learning method can be pre-trained, updated 
online or a combination of the two. Learning methods and classification are further described in Section 3.4. For thermal infrared imagery, the learning method approach is often limited by the amount of training data since few extensive training datasets exist. Transfer learning from visual imagery examples of the object can be an option (Kieritz et al. (2013)).

In the case of manual inspection of large amounts of data, automatic detection of objects can improve detection results and reduce workloads for humans. Humans are easily distracted while computers are not, making missed detections of true samples less probable. On the other hand, automatic detection might increase the amount of false alarms, depending on the object to be detected. Paper 9 describes detection of media and energy leakages in district heat networks. Thousands of images are collected for one city using an airborne thermal camera and a set of possible leakages is automatically detected and presented to the network owner. The detection algorithm follows a classic anomaly based scheme. The distribution of pixel intensities within a 2.5 meter radius around the pipe network is found, and deviating pixels in the warm end of the distribution constitutes the set of detected leakages. Post-processing (morphological operations etc.) is applied in order to close detected areas and remove too small or too large detections. Finally, a learning based scheme is applied in order to reduce false alarms.

In Paper 8, a method for detecting rails in thermal images is presented. It exploits the known geometry of the train, the fact that the train has a fixed position and orientation relative to the rails, and makes the assumption that the ground is flat in order to limit the search space. Further, the distance between rails (in meters) is known and locally constant curvature of the rails is assumed. Given all this knowledge, look up images mapping pixels to histogram bins of possible curvatures are generated enabling fast rail detection.

\subsubsection{Detection in thermal infrared}

The main approach to detection in thermal infrared has historically been thresholding, so called hotspot detection. Thermal cameras were expensive, had low resolution and interesting objects typically appeared as points (a few pixels, or even subpixels) in the image. In addition, typical objects of interest were those that are warmer than the background because they generate kinetic energy in order to move (e.g. airborne and ground vehicles). One example is airborne target detection where the object is only a few pixels wide (assuming low resolution) and the cold atmosphere serves as background. In recent years, new application areas have emerged and today, with increasing resolution, image quality, and a different set of applications, targets often span a larger pixel area, have varying temperature, and are deformable.

Thresholding combined with post-processing (e.g. merging and splitting of blobs) is an efficient detection technique in the case of high background/object contrast, a situation more or less common depending on application. Industrial applications can, for example, provide a controlled environment more suitable for thresholding that others, e.g. surveillance applications, cannot. Below are 


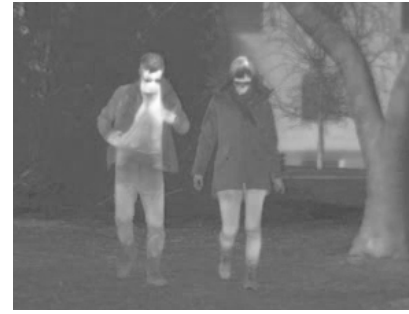

(a) Camouflage

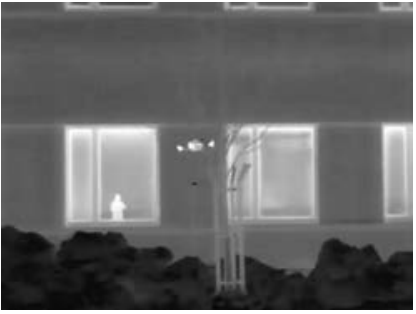

(b) Reflection

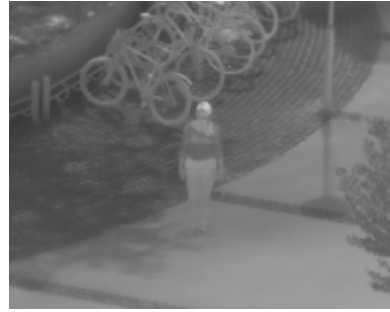

(c) Versatile background

Figure 3.2: Examples of situations where detection based on thresholding might not work.

some examples of situations where thresholding based on temperature might not work (also shown in Fig. 3.2):

Camouflage: Objects or parts of objects may have approximately the same temperature as the background. In the latter case, one object may thus give rise to several detections. For example, a human outside in the cold wearing an insulating coat. The coat will adopt the surrounding temperature, making it possible to extract only the legs and head of the object with thresholding.

Reflection: Different materials reflect thermal infrared to different extents, they have different reflectance. Wet asphalt is typically reflective as well as water puddles. Also glass has a low transmittance (and high reflectance) and will reflect most of the incoming thermal radiation. Thresholding as a detection method can not differentiate real objects from reflected ones and may thus cause false detections.

Versatile background: There might be background or other objects in the scene that have a similar appearance/intensity as the object we are interested in. Thresholding based on intensity will then give rise to false detections.

Humans try to maintain a constant body temperature which is favourable for detection algorithms. However, they also tend to wear insulating clothes making detection of humans in thermal infrared somewhat more challenging. The face of a human is typically not covered by clothes unlike the rest of the body and might therefore be easier to extract from an image. Zin et al. (2007) and Wong et al. (2010) exploit this fact by thresholding and incorprating shape information for human detection.

Some detection methods exploit the advantages of the visual and thermal modality respectively by combining information extracted from visual and thermal imagery of the same scene (Hwang et al. (2015); Apatean et al. (2010); Krotosky and Trivedi (2008)).

It is also possible to affect the temperature of the object in order to find anoma- 
lies by observing how the heat is transferred through the material. Within the field of Non-Destructive Testing (NDT), an excitation source such as a flash lamp can be used to heat the material. The process of cooling down is recorded with a high-end thermal infrared sensor. Anomalies in the material will result in anomalous heat transfer which can be detected by observing the surface. Runnemalm et al. (2014) use the described technique to detect defective spot welds.

\subsection{Tracking}

Tracking can be defined as finding the trajectory of an object over time. The trajectory is represented either by the object positions or as the complete region that the object encompasses in each observation. That is, the output consists of a state vector that can have a variable number of dimensions for different tracking methods. The choice of number of dimensions depends on the area of application and level of post-processing. The complete object region is, for example, typically required for object classification while virtual fences (intrusion alarms) only require object positions. An object is anything that might be of interest for further analysis, e.g., humans, vehicles, animals, or candy bars.

Detection and tracking are often described as two separate problems. However, tracking is dependent on detection and the task of detection can either be done separately or jointly. In the case of separately performed detection, tracking is reduced to the task of establishing correspondence between detections, to associate detections. If detection is done jointly (as in the case of e.g. template-based tracking), an initial detection is needed in order to initialize the object model. The tracking method then performs repeated specific detection. The initial detection can be found either manually or automatically. In the second case, a framework for deciding when to initialize a new instance of the tracker is needed.

Tracking can either be short- or long-term, single- or multi-sensor, as well as single- or multi-object. In this thesis, focus lies on short-term, single-object tracking using a thermal infrared sensor. Short-term tracking means both that only short periods of time, a couple of minutes maximum, are considered, and also that there is no requirement of re-identification of targets.

The main challenges with visual tracking, that is, tracking using single or multiple cameras, have been listed below (Yilmaz et al. (2006); Kristan et al. (2013); Wu et al. (2015)):

- Loss of information caused by projection of the 3D world on a 2D image (multi-camera or multi-sensor systems can help with depth information).

- Complex and unpredictable object and/or camera motion might lead to motion blur and difficulties in modelling of object motion.

- Scale variations.

- Complex object shapes.

- Nonrigid or articulated nature of objects (aspect ratio changes). 
- Partial or full object occlusions as well as self-occlusion.

- Scene illumination changes.

- Background clutter, background in the vicinity of the object has similar appearance.

- Image noise.

- Real-time processing requirements.

Common ways to reduce the complexity of the problem is to use motion and/or appearance constraints (when applicable) as well as to utilize prior knowledge about the object appearance to pre-train an object model. Tracking can also be combined with classification in order to make an online decision on which specific tracking algorithm to use. Here, the focus lies on general trackers that have the ability to adapt to any given object.

\subsubsection{Classes of tracking methods}

Tracking is a broad research area and classification of tracking methods is difficult. Primarily because of the fact that the relationship between tracking methods is complex and that hybrid trackers belonging to several categories exist. An attempt to categorise tracking methods has been made by e.g. Yilmaz et al. (2006). Another common approach is to categorise different parts of the tracking method (Smeulders et al. (2014); Wu et al. (2015)) and allowing each part to belong to several categories. The approach assumes, however, that all trackers consist of the same building blocks.

Here, a generalisation of tracking methods into two main categories has been made, point-based and appearence-based methods. The generalisation was made in order to provide a structure to the following text. The employed structure can be seen in Fig. 3.3 and each category is further described below.

\section{Point-based tracking}

Point-based tracking refers to tracking of object detections represented by points. Hence, the association of detections is made using information from the object position only, without any knowledge of the object appearance. The object state can consist of e.g. the position, velocity, and acceleration of the object. Point-based methods can be further divided into deterministic and probabilistic methods.

\section{Deterministic}

Both deterministic and probabilistic point-based tracking methods associate detections by defining and minimizing a cost for each possible association. The difference is that deterministic methods use a non-probabilistic definition of the cost, e.g., the $\ell^{2}$ norm instead of the Mahalanobis distance. Minimizing the association cost for a number of detections $N$ to a number of tracks $M$ is an optimization problem, commonly solved with the Hungarian (Auction) algorithm (Kuhn 


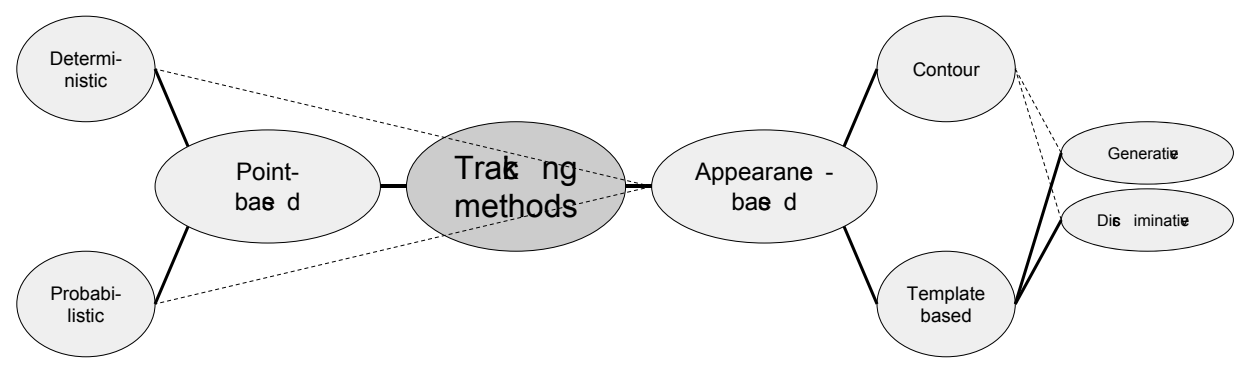

Figure 3.3: Rough categorisation of tracking methods (in order to provide a structure for the describing text). Hybrid methods exist as well. Dashed lines indicate cross-category relationships. Appearance-based tracking can, for example, also be divided into deterministic and probabilistic methods and both generative and discriminative contour tracking methods exist. There are also point-based tracking methods, so called keypoint tracking, that incorporates more information than just the object position in the object state.

and Yaw (1955)) or greedy search methods.

\section{Probabilistic}

In contrast to deterministic methods, probabilistic methods consider measurement and model uncertainties during estimation of the object state (Blackman and Popoli (1999)). They use a state space approach to model object properties, like the position, velocity, and acceleration. In the case of Gaussian distributed noise, the optimal state estimate is given by the linear Kalman filter. The Extended and Unscented Kalman filters are extensions that can handle non-linear models. The Particle filter can handle any distribution and both linear and nonlinear models (approximately), hence it is the most general state space filter mentioned here.

In the case of multi-target tracking, the association problem can be solved using one of several approaches. The simplest one is Global Nearest Neighbour (GNN). GNN associates each detection to a track, a new track, or a false alarm. Another approach is to weight all detections within a certain gating cluster using a Joint Probabilistic Data Association Filter (JPDA). This approach is favourable if the same object can give rise to several detections. The outcome of the GNN assignment problem is a hypothesis. A multi-hypothesis tracker (MHT) can keep track of several such hypotheses, not just the most likely one. It is computationally demanding but usually provides more accurate results.

The preceding text provides a brief overview of probabilistic tracking methods. Other filters and methods exist as well. The reader is referred to Blackman and Popoli (1999), Bar-Shalom and Li (1995), and Skoglar (2012) for more detailed descriptions of the methods mentioned here and more information on probabilistic tracking in general. 


\section{Appearance-based tracking}

Appearance-based tracking refers to tracking of extended objects where the object appearance plays a central role. The tracker stores a model of the object that can be continuously updated. Appearance-based methods can be further divided into contour and template-based methods.

\section{Contour}

Contours can either be tracked using shape matching or contour evolution. Both of these methods can be considered as object segmentation approaches.

Shape-matching methods store a model of the object shape, for example, an edge map or the object contour. Examples of representations are the Centroid Distance function (the distances of boundary points from its centroid), and Fourier descriptors (Granlund (1972)). More examples can be found in (Yang et al. (2008)). Another example is so called active contours (Kass et al. (1988)) that employ minimization of some energy function which can be used for shape matching.

Contour evolution approaches fit an initial contour to its new position in the current frame by either using state-space models or minimization of some energy function.

\section{Template-based}

In template-based tracking, the tracker initializes an object model by extracting an example image, a template, in the first frame. In each new frame, the tracker finds the region that best matches the template. A naive assumption, valid in certain cases, is that the object does not change and, therefore, the template does not need to be updated. In most cases, this is not true. Template-based tracking and the so called template update problem (Matthews et al. (2004)) is further described in Section 3.3.2.

Template-based tracking methods can be further divided into generative and discriminative models, the main difference being how posterior probabilities are estimated. Generative models learn the joint probability while discriminative models learn the conditional probability. Hybrid methods exist as well.

\section{Generative}

Generative tracking methods use target observations in the feature space to model a distribution of object appearances. The posterior probability of a tentative object patch being an observation of the target is typically approximated by a distance measurement in feature space from the observation to the distribution. The most well-known example of generative methods is probably the Active Appearance Models, introduced by Cootes et al. (1998).

\section{Discriminative}

Discriminative tracking methods train a classifier and tries to estimate the probability that the tentative object patch is actually an observation of the target. In contrast to generative methods, the classifier approximates the Bayesian maximuma-posteriori decision by learning the parameters of a discriminant function that 


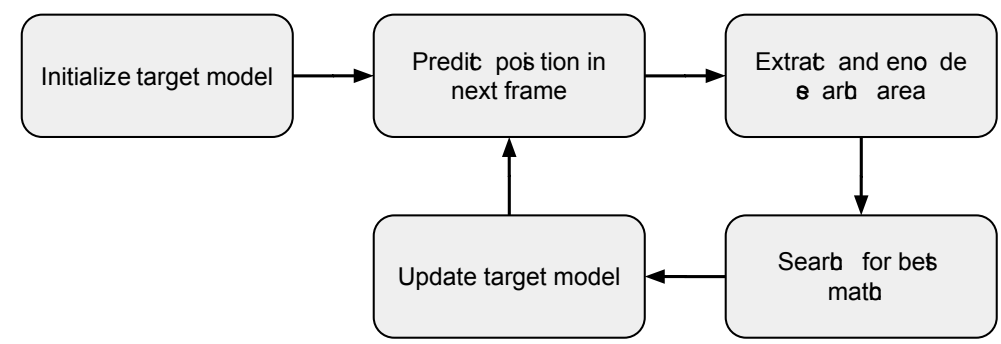

Figure 3.4: An overview of a typical template-based tracking technique, variations of the flowchart exist as well.

defines a decision boundary in a (high-dimensional) feature space.

\subsubsection{Template-based tracking}

Template-based tracking is, as was briefly described in the previous section, a tracking technique where an example image of the object, a template, is extracted in the first frame. The template constitutes an initial target model. In each new frame, the tracker finds the region that best matches the target and updates the target model. A template-based tracking method designed specifically for thermal infrared images is presented in Paper 7.

The principle of template-based tracking techniques is illustrated in Fig. 3.4. When designing a template-based tracking method, several decisions have to be made, such as the choice of a target region, a representation of appearance, a representation of motion and position, as well as a method for similarity measuring, optimization and template update. Each one of these areas are described more thoroughly below:

Target region: The most common choice for representation of the target region is the bounding box. The bounding box is advantageous due to its simple representation (position, width and height), but in most cases, it does not represent the object well. A majority of the area inside the bounding box can even be background and not object, depending on the object shape, see example in Fig. 3.5a. However, to a certain extent, including background in the target model can be beneficial since the background/object contrast can provide valuable information. Approaches where object and non-object areas inside a bounding box are found and the information incorporated in the tracking have been proposed, for example, in Paper 7 and by Ning et al. (2012). Other commonly used target regions are: ellipses, contours, blobs, patches, salient features, parts, and multiple bounding boxes (Smeulders et al. (2014)), see Fig. 3.5.

Representation of appearance: Representation of object appearance basically boils down to the question of how to represent the object pixel intensity values in order to preserve spatial and temporal information. The representation can be $\mathrm{N}$-dimensional and correspond to a $2 \mathrm{D}$-array of image data, a $1 \mathrm{D}$-histogram of 


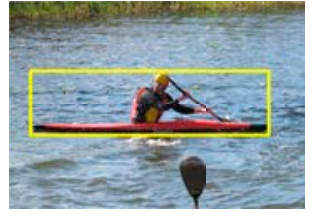

(a) Bounding box

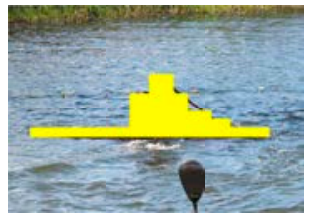

(e) Patches

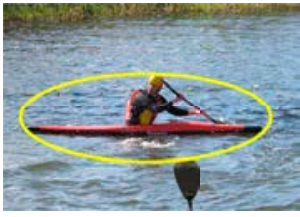

(b) Ellipse

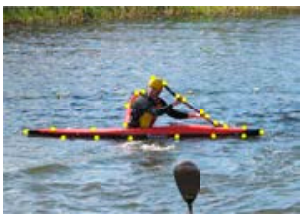

(f) Sparse set of salient features

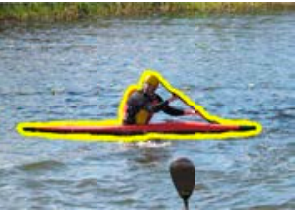

(c) Contour

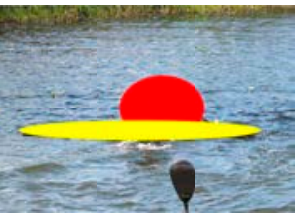

(g) Parts

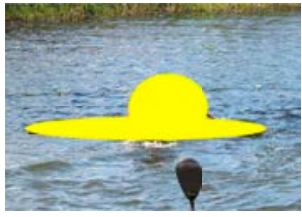

(d) Blob

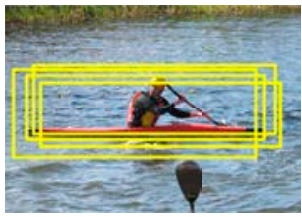

(h) Multiple

bounding boxes

Figure 3.5: Examples of target regions, here portrayed on RGB-images but the same principle applies to thermal infrared imagery as well.

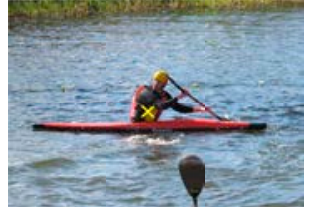

(a) Centroid

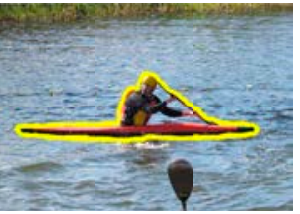

(e) Contour

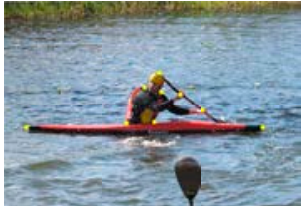

(b) Multiple control points

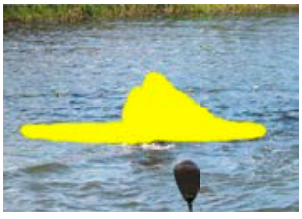

(f) Silhouette

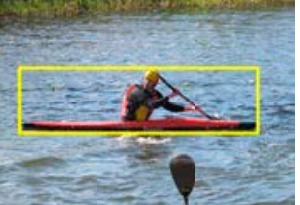

(c) Bounding box

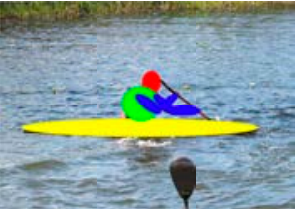

(g) Part-based, blobs

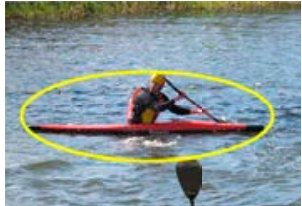

(d) Ellipse

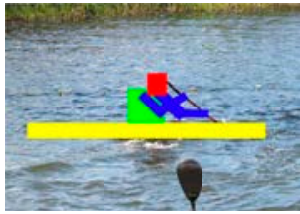

(h) Part-based, patches

Figure 3.6: Examples of different regions used for tracking annotation, here portrayed on $R G B$-images but the same principle applies to thermal infrared imagery as well. 
ordered properties, or an $\mathrm{N}$-dimensional feature vector. The simplest approach is to use the raw pixel intensity values themselves, as in, e.g., the Normalized Cross-Correlation tracker (Briechle and Hanebeck (2001)). Examples of other representations are:

- Histograms: Distribution Field Tracking (Sevilla-Lara (2012)).

- Discriminative correlation filters: CSK and DSST (Henriques et al. (2012, 2015); Danelljan et al. (2014)).

- Mixture of Gaussians.

- Kernel density estimators (Scott (1985)).

- Channel Representations (Granlund (2000)): Enhanced Distribution Field Tracking (Felsberg (2013)) and Paper 7.

Combinations of different features and representations are also possible. In addition, some trackers also consider scale variations. Optimally, information about object appearance at a larger scale should be kept. If downsampled, crucial information might be lost.

Representation of motion and position: A common choice of motion representation is to use linear or non-linear dynamic models in the form of state space models like the Kalman or Particle filters. The motion prediction is typically used to provide an initial estimate of the position in order to limit the search space. In many cases, the constant position/velocity motion model has proven to be sufficient.

Search method/similarity measurement: An initial guess for the search method is typically provided by the motion prediction. Search methods can either be exhaustive or heuristic, and are usually limited by real-time processing requirements. Heuristic methods risk being stuck in local minima.

The choice of similarity measure depends on the choice of appearance representation. Generative methods, for example, use a distance measurement that measures the distance from a tentative patch to the target distribution in feature space. Another example are methods based on correlation filters that search for the best matching patch and measures similarity via convolution.

Template update: A naive assumption is that the target appearance remains the same throughout the entire sequence. This assumption can be valid for short periods of time, but eventually, due to changes in viewpoint and lightning as well as non-rigid object motion, the initial target model will generally not be an accurate representation of the object appearance. How to correctly update the target model is one of the greatest challenges within template-based tracking. The so called template update problem has been described by, e.g., Matthews et al. (2004). It implies that small errors that are merged into the target model during update accumulates over time, causing the tracker to drift. 
The choice of update strategy typically depends on the choice of target region as well as the representation of appearance. A common approach is to use a simple linear adaptive update scheme where the object model at time $t, m^{t}$, is updated by weighting together the object model at time $t-1, m^{t-1}$ and the best matching object patch $z^{t}$ as

$$
m^{t}=(1-\alpha) m^{t-1}+\alpha z^{t}
$$

using a weight $\alpha \in[0,1]$. The model $m$ and patch $z$ are all in the chosen representation. Non-linear weighting approaches exist as well. The problem with adaptive update strategies is that they have a quite short memory span. Other update strategies that use learning methods, i.e. discriminative tracking techniques, can have longer memory spans.

\subsubsection{Tracking metrics}

Tracking performance is assessed by tracking metrics. The problem is that there is currently no consensus on what measures should be preferred. Various authors use different metrics and many variations of the same metrics exist, complicating cross-paper comparison of trackers. In addition, correlation among multiple performance measures might lead to biased tracking results towards certain tracking aspects (Čehovin et al. $(2014,2015)$ ).

Many of the papers addressing performance evaluation in visual tracking are focused on multi-target tracking scenarios, e.g., Brown et al. (2005), Edward et al. (2009), and Leichter and Krupka (2013). For single-target tracking, initiatives have been made during recent years (Čehovin et al. (2014, 2015); Kristan et al. (2013, 2014, 2015, 2016); Smeulders et al. (2014); Wu et al. (2013, 2015); Li et al. (2016)), which have led to an increased popularity for some measures that are now widely used in the literature. However, none of them has become standard. Theoretically, single target tracking is a special case of multi-target tracking, but when it comes to evaluation, there is a crucial difference in focus. In multi-target tracking, the focus lies on the accuracy of the detections, occlusion handling, and the correctness of target identity assignments. Further, evaluation is often performed on a specific application. Evaluation of single-target tracking, on the other hand, focuses on the generality, robustness, and accuracy of the tracker. The tracker has to perform well on a wide range of challenging scenarios, e.g. deformable objects, various lightning conditions, camera motion etc.

Most performance measures aim at providing an, as accurate as possible, description to what extent the predicted state of the tracker $\Lambda_{T}$ agrees with the ground truth annotation $\Lambda_{G}$. An object state $\Lambda$ at time $t$ consists of a center position $x_{t}$ and an object region $A_{t}$ :

$$
\Lambda=\left\{A_{t}, x_{t}\right\}_{t=1}^{N} .
$$

Most performance measures thus rely on ground truth annotation, a tedious and time-consuming task that also limits the use of evaluation sequences to those 
that are available with annotations. However, evaluation of tracking performance without ground truth annotations exist as well (Wu et al. (2010)).

The annotated region is typically an axis aligned bounding box, the upright rectangle with the smallest area that encloses the object. Other, more complex, regions exist as well, see examples in Fig. 3.6. What kind of region that should be chosen for an evaluation metric depends on the target region of the evaluated trackers.

In order to correctly asses the performance of a tracking algorithm many factors have to be taken into consideration. For example, robustness (how often does the tracker lose track of the target?), accuracy (how accurately is the tracking performed?), algorithm speed, memory requirement, how simple the method is to use, and how to combine these in order to appoint a winner. Further, it is important to consider what tracking algorithm parameter values to use during evaluation since the choice may have a significant influence on the results. The VOT committee has solved this problem by allowing all authors to tune and run the tracker on the benchmark dataset themselves (Kristan et al. (2015)).

Below, different types of errors and the most commonly used performance measures for single target tracking are described.

\section{Error types}

Track failure: A track failure is the most general error type. It occurs when the tracker completely misses to locate the target. It might be a consequence of drift and can be of either type false negative or false positive.

Drift: The tracker can slowly move off the target leading to decreased accuracy, or even worse, track failure. It can occur, e.g., due to accumulation of small errors in tracker prediction or tracking the background instead of the target.

False positive/detection of non-object as object: A track failure due to association with a false positive detection occurs when the tracker has found an erroneous match for the target.

False negative/missed detection: A track failure due to a missed detection occurs when the tracker cannot find a match for the target even though the target is present in the scene.

ID-switch: An ID-switch error is only relevant in the case of multiple identities, i.e., multi-target tracking. It occurs when the identity assignment of targets fails and the identities of different objects are confused.

\section{Single target tracking performance measures}

Some of the most common performance measures for single target tracking performance evaluation are described below. 


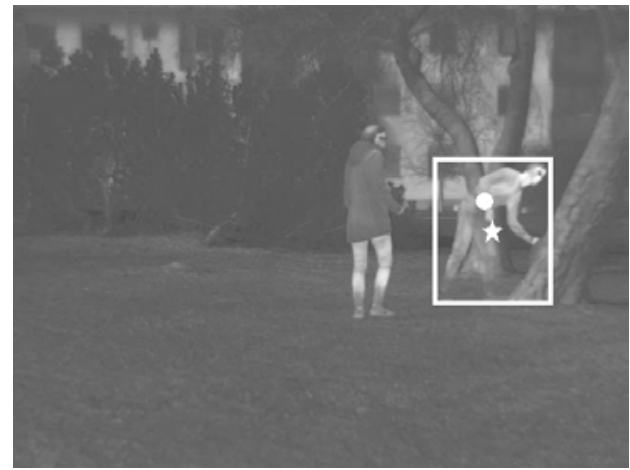

Figure 3.7: An illustration of how the center localization error is affected by the choice of target region. Here, the tracker outputs an enclosing bounding box and the center of the object is approximated by the center of the bounding box (star) which is not true in this case. The true center of the object is marked with a circle.

Center localization error: The center localization error measure is defined as the Euclidean distance between the target's predicted center $x_{t}^{T}$ and its ground truth annotation $x_{t}^{G}$ (Čehovin et al. (2015)):

$$
\Delta\left(\Lambda_{G}, \Lambda_{T}\right)=\left\{\delta_{t}\right\}_{t=1}^{N}, \quad \delta_{t}=\left\|x_{t}^{G}-x_{t}^{T}\right\|
$$

It is popular due to the minimal annotation effort, only one point per object/frame needs to be annotated. For point targets, the measure works well, but for extended targets, the measure becomes sensitive to subjective annotation. Depending on the choice of target region, the center of the region might not be consistent with the center of the object, see example in Fig. 3.7. The center error is usually summarized as an average or root-mean-square-error over all frames $N$ where the object is visible, that is

$$
\bar{\Delta}\left(\Lambda_{G}, \Lambda_{T}\right)=\frac{1}{N} \sum_{t=1}^{N} \delta_{t}
$$

or

$$
\operatorname{RMSE}\left(\Lambda_{G}, \Lambda_{T}\right)=\sqrt{\frac{1}{N} \sum_{t=1}^{N} \delta_{t}^{2}}
$$

If the tracker drifts off the target, the center localization error will become extremely large. Therefore, it is common to estimate tracking performance by using a ratio of correctly tracked frames. Correctly tracked frames are frames in which the estimated localization error is within a certain threshold. 


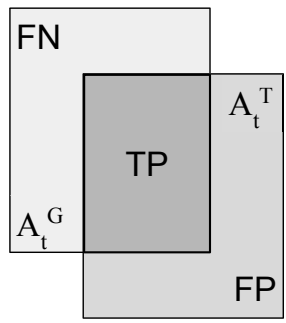

Figure 3.8: Illustration of the region overlap measure.

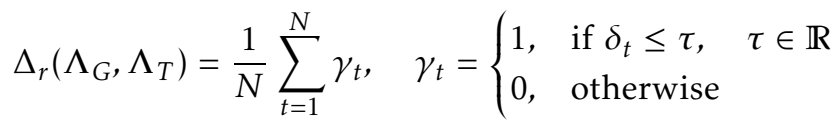

Region overlap: The region overlap measure is defined as the overlap between the target's predicted region and the ground truth region:

$$
\Phi\left(\Lambda_{G}, \Lambda_{T}\right)=\left\{\phi_{t}\right\}_{t=1}^{N}, \quad \phi_{t}=\frac{A_{t}^{G} \cap A_{t}^{T}}{A_{t}^{G} \cup A_{t}^{T}}=\frac{T P}{F N+T P+F P} .
$$

An illustration of the region overlap is provided in Fig. 3.8. In contrast to the center error localization measure, the region overlap takes both position and size into consideration and does not result in extremely large errors at tracking failures, since $\phi_{t}=0$ as soon as the tracker drifts off the target. The region overlap is usually summarized by an average overlap

$$
\bar{\Phi}\left(\Lambda_{G}, \Lambda_{T}\right)=\frac{1}{N} \sum_{t=1}^{N} \phi_{t}
$$

or as a ratio of correct tracked frames

$$
\Phi_{r}\left(\Lambda_{G}, \Lambda_{T}\right)=\frac{1}{N} \sum_{t=1}^{N} \gamma_{t}, \quad \gamma_{t}=\left\{\begin{array}{ll}
1, & \text { if } \phi_{t} \leq \tau, \\
0, & \text { otherwise }
\end{array} \quad \tau \in[0,1]\right.
$$

Tracking length: The number of successfully tracked frames from the tracker initialization to the first tracking failure is known as the tracking length. The measure addresses the failure rate of the tracker, but exploits only the first part of the sequence up to the first failure. The measure requires a definition of a tracking failure. A failure can be a decision of manual inspection or, e.g., when the region overlap is below a certain threshold as in (3.16). 
Failure rate: The failure rate represents the number of failures during a sequence. The measure addresses the drawbacks of the tracking length measure by reinitializing the tracker after each failure.

The VOT challenges (Kristan et al. (2013, 2014, 2015)) mainly use two measures known as accuracy and robustness. Tracker accuracy is a based on the region overlap measure. The overlap is averaged over a subset of valid frames. If a tracker fails, it is reinitialized, and a few frames before and after the reinitialization are not considered as valid. The robustness of a tracker is a based on the failure rate. The average number of per-sequence failures is calculated for a subset of valid frames (same definition as above). The VOT2015 challenge introduced a novel performance measure that combines accuracy and robustness by averaging the average overlaps for different sequence lengths. The measure is known as expected average overlap and was used to select a winner of the challenge (Kristan et al. (2015)).

The OTB benchmarks (Wu et al. $(2013,2015)$ ) present tracking results in precision and success plots. Precision plots are based on the center localization error and show the percentage of frames in which the estimated location is within a certain threshold (3.13). The success plot measures the region overlap ratios of each frame for certain thresholds (3.16). In both plots, the ratios are varied from 0 to 1 . In order to rank the tracking algorithms, the Area Under Curve (AUC) is calculated for each plot.

Other measures and variations of the ones described above are described in (Čehovin et al. (2014, 2015); Smeulders et al. (2014); Wu et al. (2013, 2015); Li et al. (2016)).

\subsubsection{Tracking benchmarks and datasets}

In the past two decades, visual tracking has attracted significant attention, with all right, because it is a challenging problem (Gavrila (1999); Moeslund et al. (2006); Li et al. (2013)). Approximately 40 motion or tracking papers are accepted in high profile conferences annually. This large amount of publications combined with the lack of established methodology for performance evaluation have made it difficult to assess the advances within the field. Therefore, several initiatives have been made to establish a common ground in tracking performance evaluation, such as PETS (Young and Ferryman (2005)), the Visual Object Tracking (VOT) challenges (Kristan et al. $(2013,2014,2015,2016))$ and the Object Tracking Benchmark (Wu et al. (2013, 2015)). Within the area of thermal infrared tracking, far fewer benchmarks and datasets exist. Those that do are described below.

\section{Benchmarks}

Challenges on tracking in thermal infrared imagery have, to the best of the author's knowledge, been organized on three occasions. Two of them, organized 
in 2005 and 2015, were launched in conjunction with the series of workshops on Performance Evaluation of Tracking and Surveillance (PETS). The challenge was detection, multi-camera/long-term tracking and behavior (threat) analysis in contrast to the third challenge, VOT-TIR (Felsberg et al. (2015)), which focused on the problem of short-term tracking only.

VOT-TIR, introduced in 2015, was the first thermal infrared, short-term tracking challenge. Like the VOT challenge, the VOT-TIR challenge considered singlecamera, single-target, model-free, causal trackers, applied to short-term tracking. That is, pre-built object models and incorporation of information from future frames were not allowed. VOT-TIR was featured as a sub-challenge to VOT2015, organized in conjunction with ICCV2015. The challenge enabled participants not only to evaluate their results on visual data, but also to benchmark their trackers on thermal infrared sequences. The VOT-TIR2015 challenge was based on the LTIR-dataset introduced in (Paper A). The main idea of VOT-TIR was to carry the idea of an established evaluation methodology to the area of thermal infrared data. The results are presented in Paper B.

\section{Datasets}

Compared to the amount of available datasets for evaluation of tracking methods in the visual domain, the number of available datasets for tracking evaluation in thermal infrared imagery is small. In addition, most of them are outdated and do not address the challenges that researches currently are trying to solve. This forces researchers to evaluate their methods on proprietary datasets, which makes it difficult to get an overview of advancements made in the field. A summary of currently available civilian thermal infrared datasets for benchmarking of tracking methods is provided in Table 3.1. Snapshots from each dataset are presented in Fig. 3.9.

The most common datasets for evaluation of thermal infrared tracking methods are the OTCBVS datasets (Davis and Keck (2005); Davis and Sharma (2007); Miezianko). The purpose is pedestrian detection and tracking as well as thermal/visual fusion (OSU Color-Thermal). They were published in 2005 and are characterized by low resolution, warm objects against cold backgrounds (i.e., easily tracked objects), and few challenging events.

Also the LITIV dataset mainly contains warm objects moving against cold backgrounds without any occlusions. The main purpose of this dataset was registration of visible and thermal infrared imagery. The included sequences are heavily compressed, resulting in severe compression artifacts. Furthermore, there is no ground truth for tracking.

The ASL-TID (Portmann et al. (2014)) dataset provides sequences simulating a thermal camera mounted on a UAV, thus including sequences recorded with a moving camera. The included sequences are of varying difficulty, high/low object resolution, cluttered backgrounds, and occlusions. The dataset is primarily designed for object detection, not tracking.

Only one of the existing datasets, the BU-TIV dataset (Wu et al. (2014)), provides high-resolution 16-bit sequences captured with a cooled sensor. The pur- 


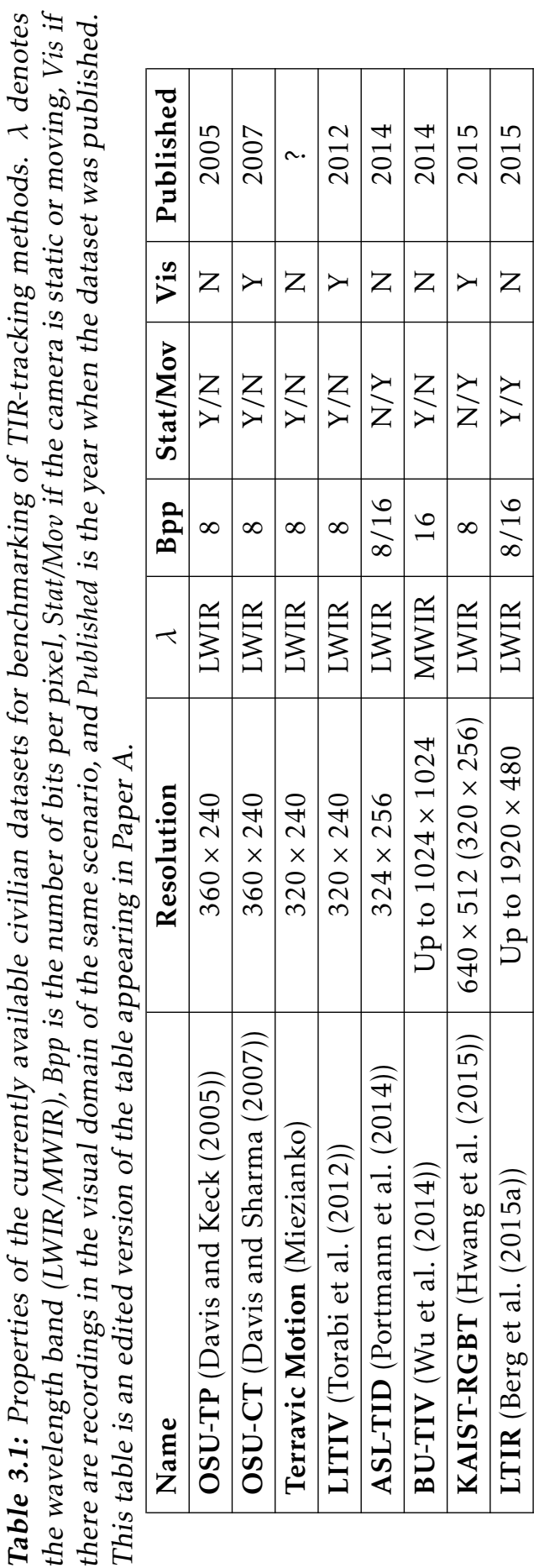


pose of the dataset is various visual analysis tasks: single-object, multiple-object, and multiple sensor tracking as well as motion pattern analysis.

KAIST-RGBT (Hwang et al. (2015)) is a multispectral pedestrian dataset which provides well aligned colour-thermal image pairs captured with a beam splitterbased hardware mounted on top of a car. Temporal correspondences for annotations are provided in order to enable evaluation of both detection and tracking methods. Thermal images are captured in $320 \times 256$ pixels, but for the registration, the image is resized to $640 \times 512$.

The Linköping Thermal Infrared (LTIR) dataset (Paper A) was designed according to the standards and rules of the VOT-challenge. That is, it contains relatively short sequences, variable difficulty and only one annotated object per sequence. Included sequences were collected from seven different sources using eight different types of sensors. They originate from industry, universities, a research institute and an EU FP7 project. Resolutions range from $320 \times 240$ to $1920 \times 480$ pixels and the average sequence length is 563 frames. Some sequences in the LTIR dataset are available with both 8- and 16-bit pixel values. There are sequences from indoor and outdoor environments, and the outdoor sequences were recorded in different weather conditions. Even though the sequences are more challenging than most sequences included in other datasets, it was still proved during the VOT-TIR challenge that an even more challenging dataset is needed.

\subsubsection{Tracking in thermal infrared}

Regarding object tracking in thermal infrared imagery, there are two common beliefs. The first is that it is all about tracking warm objects against cold backgrounds, so called hotspot tracking. This assumption is valid for certain applications only, e.g., tracking aircrafts against a cold sky, but for most other applications the situation is more complex. For example, in a surveillance application, the object can be warmer than the background initially, but as the day progresses and the sun rises and heats the surroundings, it might end up colder than the background.

The second common belief is that tracking in thermal infrared is identical to tracking in grayscale visual imagery. Consequently, a good tracker for visual imagery should also be a good tracker for thermal infrared. However, in Paper A and B it was shown that hotspot tracking is applicable for specific applications only and benchmarks were performed where trackers gave different results on RGB and thermal infrared sequences respectively.

\section{Differences between tracking in thermal infrared and visual imagery}

The differences between thermal and visual imagery were described in Section 2.4. The described characteristics of thermal infrared imagery indicate that tracking in thermal imagery is not identical to tracking in grayscale visual imagery. First, a tracker that depends heavily on (high resolution) spatial structure is presumably suboptimal for thermal infrared imagery since such imagery has a different 


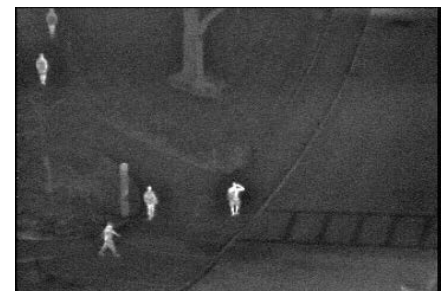

(a) OSU-TP

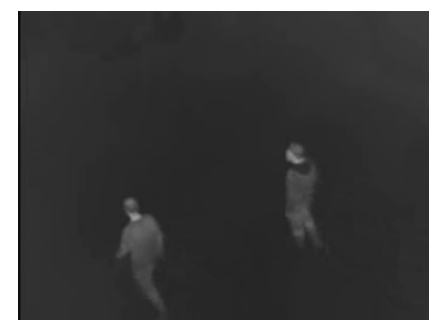

(d) LITIV

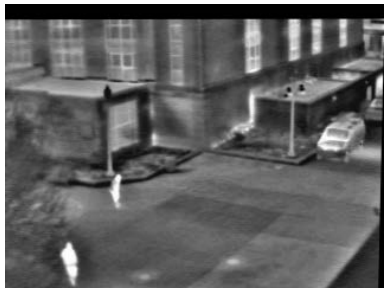

(b) OSU-CT

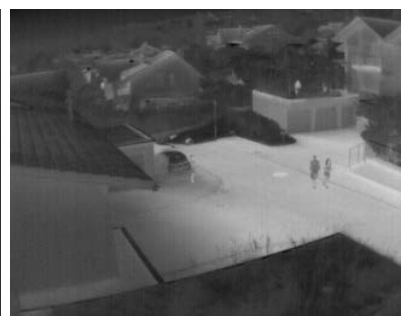

(e) ASL-TID

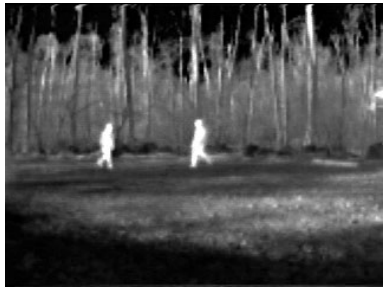

(c) Terravic Motion

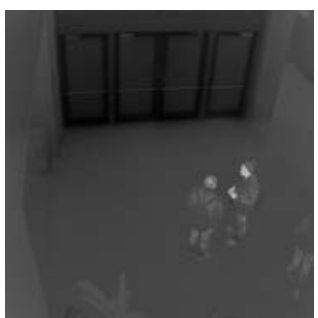

(f) $B U-T I V$

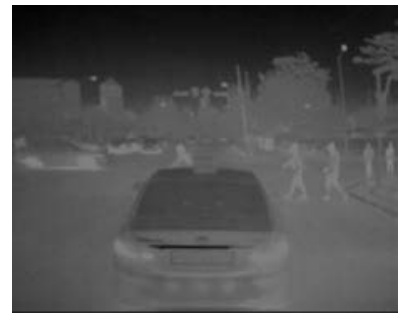

(g) KAIST-RGBT

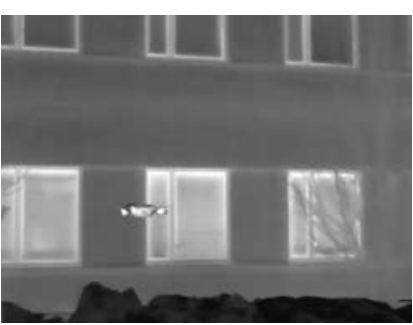

(h) LTIR

Figure 3.9: Snapshots from all datasets listed in Table 3.1. 
noise characteristics, e.g., lower resolution and more blooming.

Second, the absence of shadows might make a tracker designed to handle shadows suboptimal for thermal infrared imagery. Third, visual colour patterns are discernible in the thermal infrared spectrum only if they correspond to variations in material or temperature. Moreover, re-identification and resolving occlusions might need to be done differently. For example, two persons with differently patterned or coloured clothes might look similar in thermal infrared imagery. Fourth, trackers that exploit the absolute levels (for example, distribution field trackers) should have an advantage in thermal infrared imagery due to the fact that the emitted radiation change much slower than the reflected radiation in most applications. Fifth, trackers that are able to exploit 16-bit data from radiometric cameras should have an advantage since they have a dynamic range large enough to accommodate relevant temperature intervals without adapting the dynamic range to each frame.

Finally, perhaps the most obvious one, there is no colour in thermal infrared imagery and trackers relying on colour features are, therefore, not suitable for thermal infrared imagery. Video-rate multispectral thermal cameras exist but they are rare and out of scope of this thesis. If all these specifics mentioned above are considered, it should be possible to design a tracker superior on thermal infrared data.

\section{Benchmark results}

Benchmarking of tracking methods designed for visual imagery on both thermal and visual data have recently been performed in Paper A and B. In Paper A, seven different trackers were evaluated on the VOT 2014 (Kristan et al. (2014)) and LTIR (Berg et al. (2015a)) datasets respectively. The VOT toolkit ${ }^{2}$ was used to perform the evaluation. The evaluation indicates that different methods are optimal for visual and thermal tracking respectively. Trackers based on spatial structure and/or sparse representations are ranked better on visual imagery than they are on thermal imagery. For a tracker based on distribution of pixel values, it is the other way around.

In the VOT-TIR2015 challenge (Felsberg et al. (2015)), 24 trackers have been benchmarked on thermal infrared sequences. Evaluation was performed using the same toolkit as in VOT2015 (Kristan et al. (2015)). In addition to the VOT-TIR dataset specific analysis, an evaluation on the differences in ranking of trackers between VOT2015 and VOT-TIR2015 has been presented, see Fig. 3.10, for all common trackers. The dashed lines are the margin of a rank-change by more than three positions. Any change of rank within this margin is considered insignificant and only 8 trackers out of 21 change their rank by more than three positions.

In the VOT-TIR2015 benchmark, it was difficult to identify a systematic correlation between improvement and type of tracking methods. It is important to remember that the different challenges were conducted on different datasets with different properties (except from the spectral difference). VOT-TIR had, for example, smaller targets (in pixels) in general than VOT. One conclusion that could be

\footnotetext{
${ }^{2}$ https://github.com/vicoslab/vot-toolkit
} 


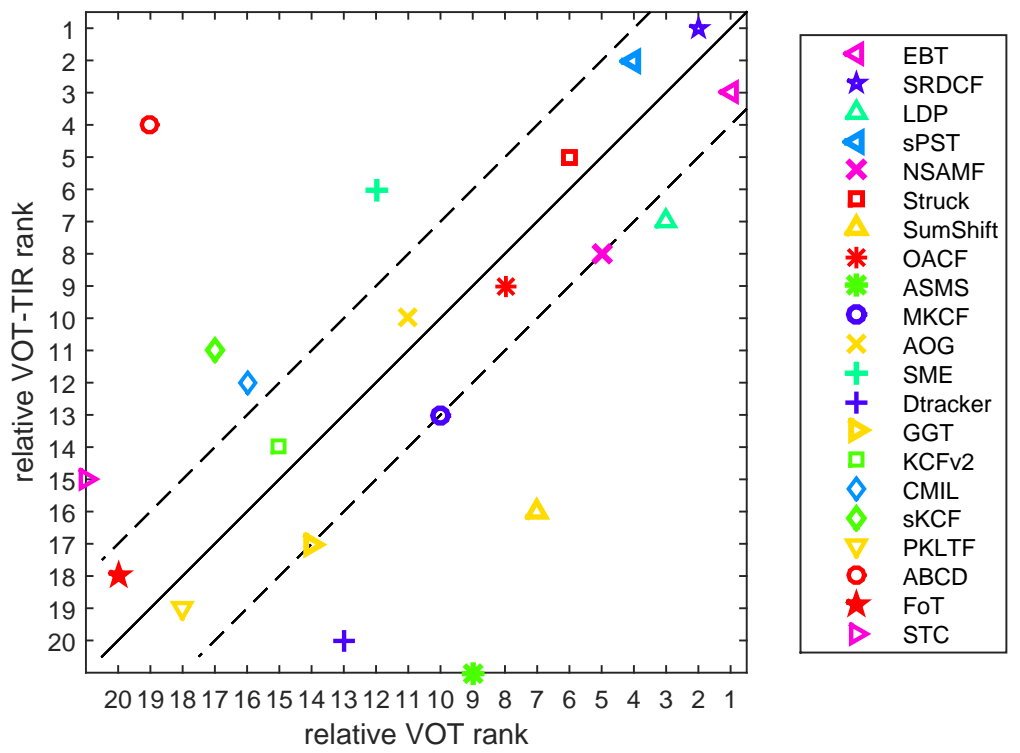

Figure 3.10: Comparison of relative ranking of 21 common trackers in VOT2015 and VOT-TIR2015.

drawn is that tracking methods that do not use colour are likely to perform better on thermal infrared sequences than colour-based methods, e.g., ASMS, SumShift, and DTracker.

\subsection{Classification}

Classification is the technique of identifying to which one of a pre-defined set of categories, or classes, a new observation belongs. It is an instance of machine learning methods where the output of the algorithm is a discrete number, a class label. In the case of a continuous output, we usually refer to regression instead. Paper 9 addresses the problem of false alarm reduction among automatically detected potential district heating leakages by employing classification methods.

\subsubsection{Feature selection}

The basic assumption of a classification problem is that distinguishing attributes, or features, between classes do exist. Features are commonly represented as $N$ dimensional feature vectors that describe different properties of the observation. In addition to being discriminative, feature vectors should also be invariant to nuisance parameters, such as illumination, small shifts in position, and scale of the region. Depending on the input imagery, features can be spatial, radiometric, spectral (colour), or temporal. When not explicitly stated otherwise, image 
features discussed here are spatial.

Due to the reasons explained above, feature selection is crucial. Features can be hand-picked or automatically learnt. Learnt features require large amounts of training data and are computationally expensive. The DeCAF features (Donahue et al. (2013)) are examples of pre-trained feature sets. Examples of common, hand-tuned features are:

- Interest point features

- Continuous: SIFT (Lowe (2004)), SURF (Bay et al. (2008)), and Channel coded feature maps (Jonsson and Felsberg (2009)).

- Binary: Local Binary Pattern (LBP) (Ojala et al. (1996)), Random ferns (Ozuysal et al. (2007)), BRIEF (Calonder et al. (2010)), and its extensions BRISK (Leutenegger et al. (2011)), FREAK (Ortiz (2012)), and ORB (Rublee et al. (2011)).

- Filter bank features: Gabor Jets (Lee (1996)), GIST (Oliva and Torralba (2001)), and Quadrature filters (Granlund and Knutsson (1995)).

- Colour histogram features: Colour names (van de Weijer et al. (2009)).

- Shape features: MSER (Matas et al. (2002)), Contour SIFT (Forssén and Lowe (2007)), Shape Context Descriptor (Belongie et al. (2002)), Fourier descriptors (Granlund (1972)).

- Others: Histograms of Oriented Gradients (HOG's) (Dalal and Triggs (2005)), Haar-like features (Viola and Jones (2001)), and Channel vectors (Granlund (2000)).

In Paper 9, an initial set of features was chosen by visual inspection of the data. The set was then reduced by a feature selection technique based on the Mahalanobis distance, $m$ :

$$
m_{f}=\sqrt{\left(\mu_{f, 1}-\mu_{f, 2}\right)^{T} S_{f}^{-1}\left(\mu_{f, 1}-\mu_{f, 2}\right)} .
$$

The technique assumes Gaussian distribution and conditional independence among samples. The Mahalanobis distance is calculated for each pair of classes and feature $f . \mu_{f, 1}$ and $\mu_{f, 2}$ are the mean values of two class distributions and $S_{f}$ is the covariance matrix of the second distribution. The approach is a common choice for measuring the "goodness" of a feature (Jain and Zongker (1997)), since the probability of error, $P_{e}$, decreases as the Mahalanobis distance increases (Duda et al. (2001)).

\subsubsection{Classification methods}

Classification methods can either be supervised or unsupervised. Supervised methods require access to labelled training examples, while unsupervised methods try to structure the data themselves by clustering. The most important property of a classification method is its ability to generalize. The problem of overfitting arises if the method has become too adapted to the training data. 
Classification can either be linear or non-linear. By a non-linear transformation of the data, it may be possible to find a new space where the classes are linearly separable, a technique known as the kernel trick.

\section{Memory based learning}

Memory-based learning techniques store object examples from different views. When classifying an observation, the observation is matched against all stored samples in memory. Hence, efficient indexing and clustering techniques are important.

\section{Learning methods}

Learning-based classification methods learn the parameters of a discriminant function that defines a decision boundary in a (high-dimensional) feature space. Examples of methods are: Support Vector Machines (SVM's) (Cortes and Vapnik (1995)), Boosting techniques (e.g. AdaBoost) (Freund and Schapire (1997)), Random forests (Breiman (2001)), artificial neural networks (McCulloch and Pitts (1943)), and its extension convolutional neural networks (CNN's) (Rumelhart et al. (1986)). Overviews of the methods mentioned above can be found in (Bishop (2006); Hastie et al. (2008)).

An online classification method is preferable if large amounts of labelled training data is not available. It can update its internal parameters and adapt the model to previously unseen views of the object, one observation at a time.

\subsubsection{Classifier evaluation}

When evaluating classification methods, it is important to separate training and testing data in order to avoid biased results. A popular evaluation method employing separate sets is $k$-fold cross-validation. The method divides the data into $k$ number of folds and performs $k$ evaluations, where each one of the folds is used for testing and the other $k-1$ for training. An illustration is provided in Fig. 3.11.

Common choices of metrics and visualization methods for classification results are Receiver Operating Characteristics (ROC) curves, Precision Recall (PR) curves, and/or confusion matrices. Confusion matrices do not only provide information about correctly and incorrectly classified samples, but also information on to which class the incorrect classification was made. The principle of a confusion matrix is visualized in Fig. 3.12 and the different types of classification outcomes are explained further below:

True positive (TP): Correct classification of positive samples.

True negative (TN): Correct classification of negative samples.

False positive (FP): Incorrect classification of negative samples as positive samples, equivalent to false alarms. 


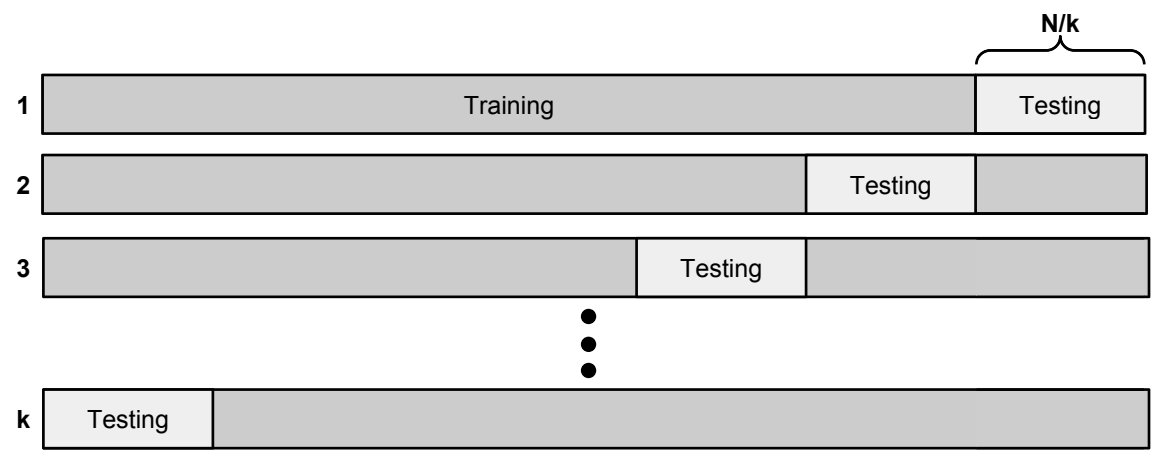

Figure 3.11: Illustration of $k$-fold cross-validation.

False negative (FN): Incorrect classification of positive samples as negative samples, equivalent to missed detections.

In addition, certain metrics can be derived from a confusion matrix. Some examples are given below:

True positive rate/Sensitivity/Recall (TPR):

$$
T P R=\frac{T P}{T P+F N}
$$

True negative rate/Specificity (TNR):

$$
T N R=\frac{T N}{F P+T N}
$$

False positive rate (FPR):

$$
F P R=\frac{F P}{F P+T N}=1-T N R
$$

Precision (PPV):

$$
P P V=\frac{T P}{T P+F P}
$$




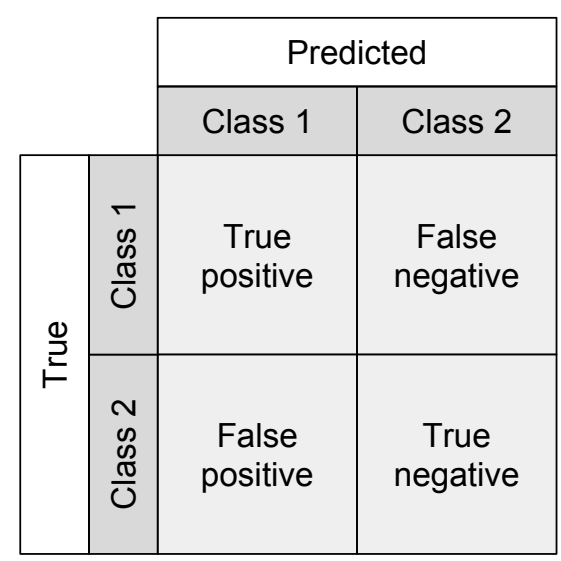

Figure 3.12: The principle of a confusion matrix. Correct classification of samples belonging to class 1 yields true positive samples, incorrect classification of samples belonging to class 1 as samples belonging to class 2 results in false negative samples, correct classification of samples belonging to class 2 yields true negative samples, and, finally, incorrect classification of samples belonging to class 2 as samples of class 1 results in false positive samples.

Accuracy (ACC):

$$
A C C=\frac{T P+T N}{T P+T N+F P+F N}
$$

F1 score:

$$
F 1=\frac{2 T P}{2 T P+F P+F N}
$$

ROC-curves measure the true and false positive rates of classification methods for different parameters. PR-curves are similar but use the precision and recall metrics instead. It is also possible to measure the AUC, area under curve. Examples of a ROC- and PR-curve are provided in Fig. 3.13. 

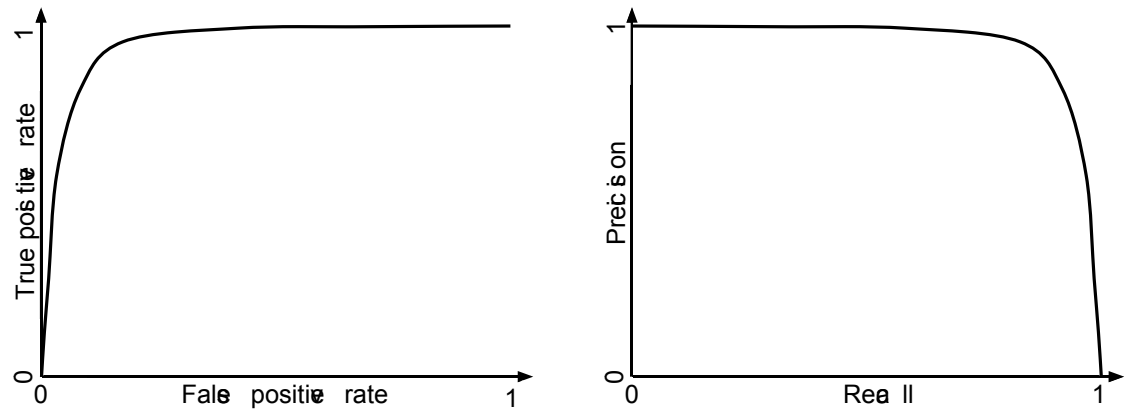

Figure 3.13: Example of a ROC-curve (left) and a PR-curve (right). 


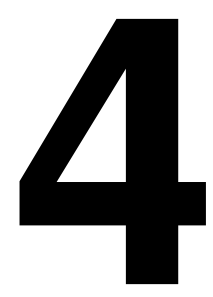

\section{Concluding Remarks}

In Part I, the background of the material included in this thesis has been provided. Chapter 1 motivated the research and presented the included publications. Chapter 2 gave an introduction to the principles of thermal infrared imaging and its applications. Detection, tracking, and classification were briefly described in Chapter 3 and specified for the specific case of thermal infrared imagery.

The results are presented in the papers in Part II. Discussions from papers are summarized in Section 4.1 and conclusions and ideas for future research are provided in Section 4.2.

\subsection{Discussion}

Papers A, B, and 7, all addressed the area of visual object tracking in thermal infrared imagery. Paper A introduced a dataset, LTIR, that was also used in a benchmark in Paper B. Both Paper A and B evaluated short-term tracking methods on thermal infrared imagery, the latter being more extensive. Paper A concluded that the available datasets at that time had become outdated, a conclusion that motivated a new dataset. The results from the VOT-TIR2015 challenge made it clear, however, that the LTIR dataset needs to be extended even further. The best performing tracker of the challenge had an average number of failures of 0.58 on 20 sequences. Hence, the LTIR dataset should be extended in order to become larger and more challenging.

Paper 7 described a tracking method for thermal infrared imagery. The method is, however, not limited to thermal data, but benchmark results showed that the proposed extensions were particularly beneficial for thermal infrared imagery. Thermal infrared imagery contains less high-resolution spatial structures, the method was, therefore, designed to exploit the absolute values of the object rather than relying on the spatial structure. A distribution field approach was utilized 
for this purpose. Background information was incorporated in the template update phase as well as in the choice of an inner bounding box, reducing the background contamination of the object model.

Two relevant applications for detection, tracking, and classification, in thermal infrared imagery were presented in Paper 8 and 9. Paper 8 proposed methods for automatic rail and obstacle detection using a train-mounted thermal camera. The rail detection method worked satisfactory but could only be used at a limited range due to its assumption of constant curvature. The combined anomaly based rail correction and obstacle detection method could successfully compensate for model errors. However, several special cases were not treated in the paper, e.g., heated railroad switches and connecting railroads. Furthermore, the evaluation of the methods was limited, a more extensive evaluation using versatile data is needed. The anomaly based obstacle detection is not limited to train-based applications, it can be used for anomaly detection in any video of approaching repetitive background. Other possible application areas include curb detection.

Paper 9 presented a classification-based approach to false alarm reduction among potential district heating leakages. The method significantly enhanced the usability of the system, reducing the number of false alarms with $58 \%$. Also, the proposed method for temporal analysis improved usability and the described visualization technique allowed the operator to get a quick overview of what areas that should be studied more carefully. Classification is in itself not a technique limited to any specific modality, however, the employed features can be. In this paper, thermographic images were used, enabling the incorporation of temperature information in the feature vectors. What features are most beneficial for thermal imagery is an area that should be studied more closely.

\subsection{Conclusions and future work}

Paper A concluded that the currently available datasets had become outdated and introduced a new dataset, LTIR. However, when the VOT-TIR2015 results were presented in Paper B, it became clear that the LTIR dataset has to be extended even further. Future work includes extending the LTIR dataset with more challenging sequences, adapting the annotation and evaluation to the current VOT standard, as well as organizing a new tracking challenge in thermal infrared imagery employing the extended dataset.

Both Paper A and Paper B evaluated the effect of the differences between RGB and thermal infrared sequences on the ranking of the trackers. Paper A concluded that trackers based on spatial structure and/or sparse representations (ASLA, DSST, SCM) are ranked better on visual imagery than they are on thermal imagery. For the tracker based on distribution of pixel values (EDFT), it was the other way around. Paper B, on the other hand, concluded that top performers in VOT-TIR2015 belonged to several different classes and that a systematic correlation between improvement and type of tracking method was difficult to identify based on the challenge results. The final conclusion of Paper B was that further evaluation of tracker performances is necessary in order to draw deeper 
conclusions about what features/principles are beneficial for thermal tracking.

Paper 7 compared trackers based on spatial structure features with trackers based on distribution fields and came to the conclusion that distribution fields are more suitable for thermal infrared tracking. Since the state-of-the-art distribution field tracker (EDFT) was not able to adapt to changing object scale, a novel method was developed to achieve adaptivity. Moreover, template-based trackers were observed to have two inherent problems of background information contaminating the template of the object to be tracked; in the search phase and in the template update phase. The proposed tracking method, ABCD, mitigated both these problems by exploiting a channel coded background distribution. ABCD has been evaluated on both RGB and thermal infrared sequences and the results show that the proposed extensions are particularly beneficial for thermal infrared imagery.

The rail detection method presented in Paper 8 works satisfactory, but, as expected, can only be used at a limited range due to its assumption of constant curvature. Future and ongoing work will include further development of both the rail and obstacle detection algorithms. There are also several special cases that will need to be addressed, such as heated railroad switches and connecting railroads, that currently result in detections. In addition to that, detection of foreground objects and/or moving objects near (not on) the rails will be added to the system.

The false alarm-reduction and temporal analysis of potential district heating leakages described in Paper 9 significantly enhanced the usability of the described system.

Summarizing the conclusions from all included papers in this thesis, the final, general, conclusion is that the different characteristics of thermal infrared imagery compared to visual imagery pose different challenges for detection and tracking methods. The full implications of these differences remain to be explored, initial experiments for the case of detection and tracking have been provided in this thesis. 



\section{Bibliography}

J. Ahlberg and A. Berg. Evaluating template rescaling in short-term single-object tracking. In Advanced Video and Signal Based Surveillance (AVSS), 2015 12th IEEE International Conference on, pages 1-4, Aug 2015. doi: 10.1109/AVSS. 2015.7301745 .

J. Ahlberg, A. Berg, and N. Markuš. Multi-person fever screening using a thermal and a visual camera. In Swedish Symposium on Image Analysis (SSBA), 2015.

A. Apatean, C. Rusu, A. Rogozan, and A. Bensrhair. Visible-infrared fusion in the frame of an obstacle recognition system. In Automation Quality and Testing Robotics (AQTR), 2010 IEEE International Conference on, volume 1, pages 1-6, May 2010. doi: 10.1109/AQTR.2010.5520865.

Y. Bar-Shalom and X.-R. Li. Multitarget-multisensor tracking : Principles and techniques. Yaakov Bar-Shalom, Storrs (US), 1995. ISBN 0-9648312-0-1. Index.

H. Bay, A. Ess, T. Tuytelaars, and L. Van Gool. Speeded-up robust features (SURF). Computer Vision and Image Understanding, 110(3):346-359, June 2008. ISSN 1077-3142. doi: 10.1016/j.cviu.2007.09.014.

S. Belongie, J. Malik, and J. Puzicha. Shape matching and object recognition using shape contexts. Pattern Analysis and Machine Intelligence, IEEE Transactions on, 24(4):509-522, Apr 2002. ISSN 0162-8828. doi: 10.1109/34.993558.

A. Berg and J. Ahlberg. Classification and temporal analysis of district heating leakages in thermal images. In The 14th International Symposium on District Heating and Cooling (DHC), 2014a.

A. Berg and J. Ahlberg. Classification of leakage detections acquired by airborne thermography of district heating networks. In Pattern Recognition in Remote Sensing (PRRS), 2014 8th IAPR Workshop on, 2014b.

A. Berg, J. Ahlberg, and M. Felsberg. Classifying district heating network leakages in aerial thermal imagery. In Swedish Symposium on Image Analysis (SSBA), 2014. 
A. Berg, J. Ahlberg, and M. Felsberg. A thermal object tracking benchmark. In Advanced Video and Signal Based Surveillance (AVSS), 2015 12th IEEE International Conference on, 2015 a.

A. Berg, J. Ahlberg, and M. Felsberg. A thermal infrared dataset for evaluation of short-term tracking methods. In Swedish Symposium on Image Analysis (SSBA), 2015b.

A. Berg, K. Öfjäll, J. Ahlberg, and M. Felsberg. Detecting rails and obstacles using a train-mounted thermal camera. In Image Analysis, volume 9127 of Lecture Notes in Computer Science, pages 492-503. Springer International Publishing, 2015c.

A. Berg, J. Ahlberg, and M. Felsberg. Channel coded distribution field tracking for thermal infrared imagery. Submitted to IEEE PETS Workshop, $2016 \mathrm{a}$.

A. Berg, J. Ahlberg, and M. Felsberg. Enhanced analysis of thermographic images for monitoring of district heat pipe networks. Submitted to Pattern Recognition Letters (PRL), 2016b.

A. Berg, M. Felsberg, G. Häger, and J. Ahlberg. An overview of the thermal infrared visual object tracking VOT-TIR2015 challenge. In Swedish Symposium on Image Analysis (SSBA), 2016c.

M. Bertozzi, A. Broggi, A. Fascioli, T. Graf, and M. Meinecke. Pedestrian detection for driver assistance using multiresolution infrared vision. Vehicular Technology, IEEE Transactions on, 53(6):1666-1678, Nov 2004. ISSN 0018-9545. doi: 10.1109/TVT.2004.834878.

C. M. Bishop. Pattern Recognition and Machine Learning. Springer Science, first edition, 2006.

S. S. Blackman and R. Popoli. Design and analysis of modern tracking systems. Artech House radar library. Artech House, Boston, London, 1999. ISBN 1-58053006-0.

V. N. Boddeti, T. Kanade, and B. V. K. V. Kumar. Correlation filters for object alignment. In Computer Vision and Pattern Recognition (CVPR), IEEE Conference on, pages 2291-2298, June 2013. doi: 10.1109/CVPR.2013.297.

D. S. Bolme, J. R. Beveridge, B. A. Draper, and Y. M. Lui. Visual object tracking using adaptive correlation filters. In Computer Vision and Pattern Recognition (CVPR), IEEE Conference on, pages 2544-2550, 2010. ISBN 978-1-4244-69840 .

L. Breiman. Random forests. Machine Learning, 45(1):5-32, October 2001. ISSN 1573-0565. doi: 10.1023/A:1010933404324.

K. Briechle and U. D. Hanebeck. Template matching using fast normalized cross correlation. In Proceedings of SPIE: Optical Pattern Recognition XII, volume 4387, pages 95-102, March 2001. doi: 10.1117/12.421129. 
L. M. Brown, A. W. Senior, Y. L. Tian, J. Connell, A. Hampapur, C. F. Shu, H. Merkl, and M. Lu. Performance evaluation of surveillance systems under varying conditions. In Proceedings of IEEE PETS Workshop, pages 1-8, 2005.

M. Calonder, V. Lepetit, C. Strecha, and P. Fua. BRIEF: binary robust independent elementary features. In Proceedings on the European Conference on Computer Vision (ECCV), pages 778-792, Berlin, Heidelberg, 2010. Springer-Verlag. ISBN 3-642-15560-X, 978-3-642-15560-4.

S. Y. Chekmenev, A. A. Farag, and E. A. Essock. Thermal imaging of the superficial temporal artery: An arterial pulse recovery model. In Computer Vision and Pattern Recognition (CVPR), IEEE Conference on, pages 1-6, June 2007. doi: 10.1109/CVPR.2007.383443.

A. Colak, B. Polat, Z. Okumus, M. Kaya, L. E. Yanmaz, and A. Hayirli. Early detection of mastitis using infrared thermography in dairy cows. Journal of Dairy Science, 91(11):4244-4248, 2008.

T. F. Cootes, G. J. Edwards, and C. J. Taylor. Active appearance models. In Proceedings on the European Conference on Computer Vision (ECCV), pages 484498, 1998. ISBN 978-3-540-69235-5. doi: 10.1007/BFb0054760.

C. Cortes and V. Vapnik. Support-vector networks. Machine Learning, 20(3): 273-297, 1995. ISSN 1573-0565. doi: 10.1023/A:1022627411411.

N. Dalal and B. Triggs. Histograms of oriented gradients for human detection. In Computer Vision and Pattern Recognition (CVPR), IEEE Conference on, volume 2, pages 886-893, June 2005.

M. Danelljan, G. Häger, F. S. Khan, and M. Felsberg. Accurate scale estimation for robust visual tracking. BMVA Press, 2014. doi: http://dx.doi.org/10.5244/ C.28.65.

J.W. Davis and M. A. Keck. A two-stage template approach to person detection in thermal imagery. Applications of Computer Vision and the IEEE Workshop on Motion and Video Computing, IEEE Workshop on, 1:364-369, 2005. doi: http://doi.ieeecomputersociety.org/10.1109/ACVMOT.2005.14.

J. W. Davis and V. Sharma. Robust detection of people in thermal imagery. In Proceedings of the International Conference on Pattern Recognition, volume 4, pages 713-716 Vol.4, Aug 2004. doi: 10.1109/ICPR.2004.1333872.

J. W. Davis and V. Sharma. Background-subtraction using contour-based fusion of thermal and visible imagery. Computer Vision and Image Understanding, 106(2-3):162-182, May 2007. ISSN 1077-3142. doi: 10.1016/j.cviu.2006.06. 010.

J. Donahue, Y. Jia, O. Vinyals, J. Hoffman, N. Zhang, E. Tzeng, and T. Darrell. DeCAF: A deep convolutional activation feature for generic visual recognition. CoRR, abs/1310.1531, 2013. 
R. O. Duda, P. E. Hart, and D.G Stork. Pattern Classification. John Wiley \& Sons, second edition, 2001.

K. K. Edward, P. D. Matthew, and B. H. Michael. An information theoretic approach for tracker performance evaluation. In Computer Vision (ICCV), IEEE International Conference on, pages 1523-1529, Sept 2009. doi: 10.1109/ICCV. 2009.5459275.

M. Felsberg. Enhanced distribution field tracking using channel representations. In Computer Vision Workshops (ICCVW), IEEE International Conference on, pages 121-128, Dec 2013. doi: 10.1109/ICCVW.2013.22.

M. Felsberg, A. Berg, G. Häger, J. Ahlberg, M. Kristan, J. Matas, A. Leonardis, L. Čehovin, G. Fernandez, and et al. The thermal infrared visual object tracking VOT-TIR2015 challenge results. In Computer Vision Workshops (ICCVW), IEEE International Conference on, pages 639-651, Dec 2015. doi: 10.1109/ICCVW.2015.86.

P.-E. Forssén and D.G. Lowe. Shape descriptors for maximally stable extremal regions. In Computer Vision (ICCV), IEEE International Conference on, pages 1-8, Oct 2007. doi: 10.1109/ICCV.2007.4409025.

Y. Freund and R. E. Schapire. A decision-theoretic generalization of on-line learning and an application to boosting. Journal of Computer and System Sciences, 55(1):119-139, August 1997. ISSN 0022-0000. doi: 10.1006/jcss.1997.1504.

H. K. Galoogahi, T. Sim, and S. Lucey. Multi-channel correlation filters. In Computer Vision (ICCV), IEEE International Conference on, pages 3072-3079, Dec 2013. doi: 10.1109/ICCV.2013.381.

V. Gan, P. Carr, and J. Soltis. Monitoring giraffe behavior in thermal video. In Applications and Computer Vision Workshops (WACVW), IEEE Winter Conference on, pages 36-43, Jan 2015. doi: 10.1109/WACVW.2015.8.

D. M. Gavrila. The visual analysis of human movement: A survey. Computer Vision and Image Understanding, 73(1):82-98, 1999.

N. Goyette, P. M. Jodoin, F. Porikli, J. Konrad, and P. Ishwar. Changedetection.net: A new change detection benchmark dataset. In Computer Vision and Pattern Recognition Workshops (CVPRW), IEEE Conference on, pages 1-8, June 2012. doi: 10.1109/CVPRW.2012.6238919.

G. H. Granlund. Fourier preprocessing for hand print character recognition. Computers, IEEE Transactions on, C-21(2):195-201, Feb 1972. ISSN 00189340. doi: 10.1109/TC.1972.5008926.

G. H. Granlund. An associative perception-action structure using a localized space variant information representation. In Proceedings of the Second International Workshop on Algebraic Frames for the Perception-Action Cycle, AFPAC '00, pages 48-68, London, UK, UK, 2000. Springer-Verlag. ISBN 3-54041013-9. 
G. H. Granlund and H. Knutsson. Signal Processing for Computer Vision. Kluwer Academic Publishers, Norwell, MA, USA, 1995. ISBN 0792395301.

M. D. Gregorio and M. Giordano. Change detection with weightless neural networks. In Computer Vision and Pattern Recognition Workshops (CVPRW), IEEE Conference on, pages 409-413, June 2014. doi: 10.1109/CVPRW.2014.66.

J. Han and B. Bhanu. Human activity recognition in thermal infrared imagery. In Computer Vision and Pattern Recognition Workshops (CVPRW), IEEE Conference on, pages 17-17, June 2005. doi: 10.1109/CVPR.2005.469.

T. Hastie, R. Tibshirani, and J. Friedman. The elements of statistical learning. Springer, second edition, 2008.

J. F. Henriques, R. Caseiro, P. Martins, and J. Batista. Exploiting the circulant structure of tracking-by-detection with kernels. In Proceedings on the European Conference on Computer Vision (ECCV), pages 702-715, Berlin, Heidelberg, 2012. Springer-Verlag. ISBN 978-3-642-33764-2. doi: 10.1007/ 978-3-642-33765-9_50.

J. F. Henriques, J. Carreira, R. Caseiro, and J. Batista. Beyond hard negative mining: Efficient detector learning via block-circulant decomposition. In Computer Vision (ICCV), IEEE International Conference on, pages 2760-2767, Dec 2013. doi: 10.1109/ICCV.2013.343.

J. F. Henriques, R. Caseiro, P. Martins, and J. Batista. High-speed tracking with kernelized correlation filters. Pattern Analysis and Machine Intelligence, IEEE Transactions on, 2015. doi: 10.1109/TPAMI.2014.2345390.

L. Hoegner and U. Stilla. Thermal leakage detection on building facades using infrared textures generated by mobile mapping. In Urban Remote Sensing Event, 2009 Joint, pages 1-6, May 2009. doi: 10.1109/URS.2009.5137681.

N. Hristov, M. Betke, D. Theriault, A. Bagchi, and T. Kunz. Seasonal variation in colony size of brazilian free-tailed bats at carlsbad cavern based on thermal imaging. Journal of Mammalogy, 91(1):183-192, 2010.

S. Hwang, J. Park, N. Kim, Y. Choi, and I. S. Kweon. Multispectral pedestrian detection: Benchmark dataset and baseline. In Computer Vision and Pattern Recognition (CVPR), IEEE Conference on, 2015.

A. Jain and D. Zongker. Feature selection: Evaluation, application, and small sample performance. Pattern Analysis and Machine Intelligence, IEEE Transactions on, 19:153-158, 1997.

E. Jonsson and M. Felsberg. Efficient computation of channel-coded feature maps through piecewise polynomials. Image and Vision Computing, 27(11):16881694, 2009. 
M. Kass, A. Witkin, and D. Terzopoulos. Snakes: Active contour models. International Journal of Computer Vision, 1(4):321-331, 1988. ISSN 1573-1405. doi: 10.1007/BF00133570.

H. Kieritz, W. Hübner, and M. Arens. Learning transmodal person detectors from single spectral training sets. In Proc. SPIE, volume 8901, pages 89010F89010F-9, 2013. doi: 10.1117/12.2028651.

M. Kristan, R. Pflugfelder, A. Leonardis, J. Matas, F. Porikli, L. Čehovin, G. Nebehay, G. Fernandez, T. Vojír r, and et al. The visual object tracking VOT2013 challenge results. In Computer Vision Workshops (ICCVW), IEEE International Conference on, pages 98-111, 2013. ISBN 9780769551616. doi: 10.1109/ICCVW.2013.20.

M. Kristan, R. Pflugfelder, A. Leonardis, J. Matas, L. Čehovin, G. Nebehay, T. Vojír r, G. Fernandez, and et al. The visual object tracking VOT2014 challenge results. In Proceedings on the European Conference on Computer Vision Workshop (ECCVW), volume 8926 of Lecture Notes in Computer Science, pages 191-217. Springer, 2014.

M. Kristan, J. Matas, A. Leonardis, M. Felsberg, L. Čehovin, G. Fernández, T. Vojír G. Nebehay, R. Pflugfelder, G. Häger, and et al. The visual object tracking VOT2015 challenge results. In Computer Vision Workshops (ICCVW), IEEE International Conference on, 2015.

M. Kristan, J. Matas, A. Leonardis, T. Vojí r, R. Pflugfelder, G. Fernandez, G. Nebehay, F. Porikli, and L. Čehovin. A novel performance evaluation methodology for single-target trackers. Pattern Analysis and Machine Intelligence, IEEE Transactions on, PP(99):1-1, 2016. ISSN 0162-8828.

S. J. Krotosky and M. M. Trivedi. Person surveillance using visual and infrared imagery. Circuits and Systems for Video Technology, IEEE Transactions on, 18 (8):1096-1105, Aug 2008. ISSN 1051-8215. doi: 10.1109/TCSVT.2008.928217.

H. W. Kuhn and B. Yaw. The hungarian method for the assignment problem. Naval Research Logistics Quarterly, pages 83-97, 1955.

B. B. Lahiri, S. Bagavathiappan, T. Jayakumar, and J. Philip. Medical applications of infrared thermography: A review. Instrumentation Measurement Magazine, IEEE, 55(4):221-235, Jul 2012. doi: 10.1016/j.infrared.2012.03.007.

T. S. Lee. Image representation using $2 \mathrm{D}$ gabor wavelets. IEEE Trans. Pattern Anal. Mach. Intell., 18(10):959-971, October 1996. ISSN 0162-8828. doi: 10. $1109 / 34.541406$.

I. Leichter and E. Krupka. Monotonicity and error type differentiability in performance measures for target detection and tracking in video. Pattern Analysis and Machine Intelligence, IEEE Transactions on, 35(10):2553-2560, Oct 2013. ISSN 0162-8828. doi: 10.1109/TPAMI.2013.70. 
S. Leutenegger, M. Chli, and R. Y. Siegwart. Brisk: Binary robust invariant scalable keypoints. In Computer Vision (ICCV), IEEE International Conference on, pages 2548-2555, Nov 2011. doi: 10.1109/ICCV.2011.6126542.

A. Li, M. Lin, Y. Wu, M. H. Yang, and S. Yan. NUS-PRO: A new visual tracking challenge. Pattern Analysis and Machine Intelligence, IEEE Transactions on, 38 (2):335-349, Feb 2016. ISSN 0162-8828. doi: 10.1109/TPAMI.2015.2417577.

X. Li, W. Hu, C. Shen, Z. Zhang, A. R. Dick, and A. Van den Hengel. A survey of appearance models in visual object tracking. arXiv:1303.4803 [cs.CV], 2013.

A. Linderhed, S. Nyberg, S. Sjökvist, and M. Uppsäll. Optical methods for detection of minefields. Technical Report FOI-R-1331-SE, Swedish Defense Research Agency, January 2004.

D. G. Lowe. Distinctive image features from scale-invariant keypoints. International Journal of Computer Vision, 60(2):91-110, November 2004. ISSN 09205691. doi: 10.1023/B:VISI.0000029664.99615.94.

N. Markuš, M. Frljak, I. S. Pandzic, J. Ahlberg, and R. Forchheimer. Fast localization of facial landmark points. CoRR, abs/1403.6888, 2014. URL http: //arxiv.org/abs/1403.6888.

J. Matas, O. Chum, M. Urban, and T. Pajdla. Robust wide baseline stereo from maximally stable extremal regions. In Proceedings of the British Machine Vision Conference, pages 384-393, 2002.

I. Matthews, T. Ishikawa, and S. Baker. The template update problem. Pattern Analysis and Machine Intelligence, IEEE Transactions on, 26(6):810-815, June 2004. ISSN 0162-8828. doi: 10.1109/TPAMI.2004.16.

W. S. McCulloch and W. Pitts. A logical calculus of the ideas immanent in nervous activity. The bulletin of mathematical biophysics, 5(4):115-133, 1943. ISSN 1522-9602. doi: 10.1007/BF02478259.

Roland Miezianko. IEEE OTCBVS WS series bench; terravic research infrared database.

T. B. Moeslund, A. Hilton, and V. Kruger. A survey of advances in vision-based human motion capture and analysis. Computer Vision and Image Understanding, 103(2-3):90-126, November 2006.

Y. M. H. Ng, M. Yu, Y. Huang, and R. Du. Diagnosis of sheet metal stamping processes based on 3-D thermal energy distribution. Automation Science and Engineering, IEEE Transactions on, 4(1):22-30, Jan 2007. ISSN 1545-5955. doi: 10.1109/TASE.2006.873227.

J. Ning, L. Zhang, D. Zhang, and C. Wu. Robust mean-shift tracking with corrected background-weighted histogram. Computer Vision, IET, 6(1):62-69, January 2012. ISSN 1751-9632. 
T. Ojala, M. Pietikäinen, and D. Harwood. A comparative study of texture measures with classification based on featured distributions. Pattern Recognition, 29(1):51-59, January 1996. doi: 10.1016/0031-3203(95)00067-4.

A. Oliva and A. Torralba. Modeling the shape of the scene: A holistic representation of the spatial envelope. International Journal of Computer Vision, 42(3): 145-175, 2001. ISSN 1573-1405. doi: 10.1023/A:1011139631724.

R. Ortiz. FREAK: fast retina keypoint. In Computer Vision and Pattern Recognition (CVPR), IEEE Conference on, pages 510-517, 2012. ISBN 978-1-46731226-4.

M. Ozuysal, P. Fua, and V. Lepetit. Fast keypoint recognition in ten lines of code. In Computer Vision and Pattern Recognition (CVPR), IEEE Conference on, pages 1-8, June 2007. doi: 10.1109/CVPR.2007.383123.

R. Paugam, M. J. Wooster, and G. Roberts. Use of handheld thermal imager data for airborne mapping of fire radiative power and energy and flame front rate of spread. Geoscience and Remote Sensing, IEEE Transactions on, 51(6):33853399, June 2013. ISSN 0196-2892. doi: 10.1109/TGRS.2012.2220368.

J. Portmann, S. Lynen, M. Chli, and R. Siegwart. People detection and tracking from aerial thermal views. In Proceedings of the IEEE International Conference on Robotics and Automation (ICRA), 2014.

W.G. Rees. Physical Principles of Remote Sensing. Cambridge University Press, second edition, 2001. ISBN 9780511812903. Cambridge Books Online.

A. Rogalski. History of infrared detectors. Opto-Electronics Review, 20(3):279308, 2012. ISSN 1230-3402. doi: 10.2478/s11772-012-0037-7.

E. Rublee, V. Rabaud, K. Konolige, and G. Bradski. ORB: An efficient alternative to SIFT or SURF. In Computer Vision (ICCV), IEEE International Conference on, pages 2564-2571, Nov 2011. doi: 10.1109/ICCV.2011.6126544.

P. Rudol and P. Doherty. Human body detection and geolocalization for UAV search and rescue missions using color and thermal imagery. In Aerospace Conference, 2008 IEEE, pages 1-8, March 2008. doi: 10.1109/AERO.2008. 4526559 .

D. E. Rumelhart, G. E. Hinton, and R. J. Williams. Learning internal representations by error propagation. In Parallel Distributed Processing - Explorations in the Microstructure of Cognition, chapter 8, pages 318-362. MIT Press, 1986.

A. Runnemalm, J. Ahlberg, A. Appelgren, and S. Sjökvist. Automatic inspection of spot welds by thermography. Journal of Nondestructive Evaluation, 33(3): 398-406, 2014. ISSN 0195-9298. doi: 10.1007/s10921-014-0233-0.

J. Rydell and E. Bilock. Real-time positioning and mapping with the panther chameleon. In Swedish Symposium on Image Analysis (SSBA), 2015. 
D. W. Scott. Averaged shifted histograms: Effective nonparametric density estimators in several dimensions. The Annals of Statistics, 13(3):1024-1040, 09 1985. doi: 10.1214/aos/1176349654.

M. Sedky, M. Moniri, and C. C. Chibelushi. Spectral-360: A physics-based technique for change detection. In Computer Vision and Pattern Recognition Workshops (CVPRW), IEEE Conference on, pages 405-408, June 2014. doi: 10.1109/CVPRW.2014.65.

L. Sevilla-Lara. Distribution fields for tracking. In Computer Vision and Pattern Recognition (CVPR), IEEE Conference on, pages 1910-1917, 2012. ISBN 9781-4673-1226-4.

R. Siegel. Land mine detection. Instrumentation Measurement Magazine, IEEE, 5(4):22-28, Dec 2002. ISSN 1094-6969. doi: 10.1109/MIM.2002.1048979.

P. Skoglar. Tracking and Planning for Surveillance Applications. PhD thesis, Linköping university, 2012.

A. W. M. Smeulders, D. M. Chu, R. Cucchiara, S. Calderara, A. Dehghan, and M. Shah. Visual tracking: An experimental survey. Pattern Analysis and Machine Intelligence, IEEE Transactions on, 36(7):1442-1468, July 2014. ISSN 0162-8828. doi: 10.1109/TPAMI.2013.230.

A. Torabi, G. Massé, and G. A. Bilodeau. An iterative integrated framework for thermal-visible image registration, sensor fusion, and people tracking for video surveillance applications. Computer Vision and Image Understanding, 116(2): 210 - 221, 2012. ISSN 1077-3142. doi: 10.1016/j.cviu.2011.10.006.

K. Toyama, J. Krumm, B. Brumitt, and B. Meyers. Wallflower: principles and practice of background maintenance. In Computer Vision (ICCV), IEEE International Conference on, volume 1, pages 255-261 vol.1, 1999. doi: 10.1109/ ICCV.1999.791228.

J. van de Weijer, C. Schmid, J. Verbeek, and D. Larlus. Learning color names for real-world applications. Image Processing, IEEE Transactions on, 18(7):15121523, July 2009. ISSN 1057-7149.

L. Čehovin, M. Kristan, and A. Leonardis. Is my new tracker really better than yours? In Applications and Computer Vision (WACV), IEEE Winter Conference on, pages 540-547, March 2014. doi: 10.1109/WACV.2014.6836055.

L. Čehovin, A. Leonardis, and M. Kristan. Visual object tracking performance measures revisited. CoRR, abs/1502.05803, 2015. URL http: / / arxiv.org/ abs/1502.05803.

P. Viola and M. Jones. Rapid object detection using a boosted cascade of simple features. In Computer Vision and Pattern Recognition (CVPR), IEEE Conference on, volume 1, pages I-511-I-518 vol.1, 2001. doi: 10.1109/CVPR.2001. 990517. 
R. Wang, F. Bunyak, G. Seetharaman, and K. Palaniappan. Static and moving object detection using flux tensor with split gaussian models. In Computer Vision and Pattern Recognition Workshops (CVPRW), IEEE Conference on, pages 420-424, June 2014a. doi: 10.1109/CVPRW.2014.68.

Y. Wang, P. M. Jodoin, F. Porikli, J. Konrad, Y. Benezeth, and P. Ishwar. CDnet 2014: an expanded change detection benchmark dataset. In Computer Vision and Pattern Recognition Workshops (CVPRW), IEEE Conference on, pages 393400, June 2014b. doi: 10.1109/CVPRW.2014.126.

W. K. Wong, Z. Y. Chew, C. K. Loo, and W. S. Lim. An effective trespasser detection system using thermal camera. In Computer Research and Development, 2010 Second International Conference on, pages 702-706, May 2010. doi: 10.1109/ICCRD.2010.161.

H. Wu, A. C. Sankaranarayanan, and R. Chellappa. Online empirical evaluation of tracking algorithms. Pattern Analysis and Machine Intelligence, IEEE Transactions on, 32(8):1443-1458, Aug 2010. ISSN 0162-8828. doi: 10.1109/TPAMI.2009.135.

Y. Wu, Y. Lim, and M. H. Yang. Online object tracking: A benchmark. In Computer Vision and Pattern Recognition (CVPR), IEEE Conference on, pages 2411-2418, June 2013. doi: 10.1109/CVPR.2013.312.

Y. Wu, J. Lim, and M. Yang. Object tracking benchmark. Pattern Analysis and Machine Intelligence, IEEE Transactions on, 37(9):1834-1848, Sept 2015. ISSN 0162-8828. doi: 10.1109/TPAMI.2014.2388226.

$\mathrm{Z}$. Wu, N. Fuller, D. Theriault, and M. Betke. A thermal infrared video benchmark for visual analysis. In Computer Vision and Pattern Recognition Workshops (CVPRW), IEEE Conference on, June 2014.

M. Yang, K. Kpalma, and J. Ronsin. A survey of shape feature extraction techniques. In Peng-Yeng Yin, editor, Pattern Recognition, pages 43-90. IN-TECH, November 2008. 38 pages.

A. Yilmaz, O. Javed, and M. Shah. Object tracking: A survey. ACM Comput. Surv., 38(4), December 2006. ISSN 0360-0300. doi: 10.1145/1177352.1177355.

D. P. Young and J. M. Ferryman. PETS Metrics: On-line performance evaluation service. In ICCCN '05 Proceedings of the 14th International Conference on Computer Communications and Networks, pages 317-324, 2005.

L. Zhang, B. Wu, and R. Nevatia. Pedestrian detection in infrared images based on local shape features. In Computer Vision and Pattern Recognition (CVPR), IEEE Conference on, pages 1-8, June 2007. doi: 10.1109/CVPR.2007.383452.

T. T. Zin, R. Takahashi, and H. Hama. Robust person detection using far infrared camera for image fusion. In Innovative Computing, Information and Control, 2007. ICICIC '07. Second International Conference on, pages 310-310, Sept 2007. doi: 10.1109/ICICIC.2007.501. 
Part II

\section{Publications}





\section{Publications}

The articles associated with this thesis have been removed for copyright reasons. For more details about these see:

http://urn.kb.se/resolve?urn=urn:nbn:se:liu:diva-126955 